\title{
Recovery of a supersonic turbulent boundary layer after an expansion corner
}

\author{
Ming-bo Sun $^{1,2^{*}}$, Zhiwei $\mathrm{Hu}^{2}$, Neil D. Sandham ${ }^{2}$ \\ ${ }^{1}$ Science and Technology on Scramjet Laboratory, National University of Defense Technology, \\ Changsha, 410073, China \\ ${ }^{2}$ Faculty of Engineering and the Environment, University of Southampton, Southampton, SO17 \\ 1BJ, UK
}

\begin{abstract}
Supersonic turbulent flows at Mach 2.7 over expansion corners with deflection angles of $0^{\circ}$ (flat plate), $2^{\circ}$ and $4^{\circ}$ have been studied using direct numerical simulation. Distributions of skin friction, pressure, velocity and the boundary layer growth show that the turbulent boundary layer experiences a recovery from a non-equilibrium to an equilibrium state downstream of the expansion corner. Analysis of velocity profiles indicates that the streamwise velocity undergoes a reduction in the near-wall region even though the velocity in the core part of the boundary layer is accelerated after the expansion corner. Growth of the boundary layer was evaluated and a higher shape factor was found in the expansion cases. Turbulence was found to be mostly suppressed downstream of the corner, and throughout the recovery region, even though turbulence is regenerated in the nearwall region. The expansion ramp increases the near wall streak spacing compared to a flat plate and turbulent kinetic energy profiles and budgets exhibit a characteristic two-layer structure. Nearwall turbulence recovers to a balance between the local production and dissipation equilibrium more quickly in the inner layer than in the outer layer. The two-layer structure is due to a history effect of turbulence decay in the outer part of the boundary layer downstream of the expansion corner, with limited momentum and energy exchange between the inner layer and the main stream.
\end{abstract}

*Corresponding author: Mingbo Sun, email: sunmingbonudt@sina.cn 


\section{Introduction}

The expansion corner is a representative configuration to study the response of a compressible turbulent boundary layer (TBL) to the effects of a favorable pressure gradient. As a supersonic flow passes over an expansion corner, it is accelerated through an expansion fan and the boundary layer becomes thicker due to the decrease in density. After the expansion, the boundary layer experiences a relaxation process, during which it exhibits different turbulence features from the incoming equilibrium state. The suppression of turbulence during the expansion process is a significant characteristic of supersonic flow passing an isolated expansion corner, as reviewed by Knight et al. ${ }^{[1]}$ and could lead to boundary layer re-laminarization when the flow experiences a strong expansion.

A two-layer structure has been observed during the boundary layer expansion and recovery ${ }^{[2-4]}$, which includes a near-wall active layer, where turbulence is anisotropic and still in production, and an outer layer containing nearly isotropic "debris" inherited from the upstream boundary layer. Turbulence is suppressed due to the combined effects of the favorable pressure gradient, the convex curvature of streamlines, and the bulk dilatation. Experiments ${ }^{[5]}$ demonstrated that the absence of shear stress in the outer layer eliminates turbulence production and consequently the turbulence decays. Recently Tichenor et al. ${ }^{[6]}$ examined the effect of favorable pressure gradients on a fully developed Mach 4.9 TBL. They observed the expected stabilizing trends in the Reynolds stresses, and found that the Reynolds shear stress changes sign in the outer part of the boundary layer for the strongest favourable pressure gradient investigated. Rapid Distortion Theory ${ }^{[4,7]}$ can reproduce the reduction in the longitudinal turbulence intensity well in the outer part of the boundary layer, but provides poor predictions near the wall. Direct numerical simulations ${ }^{[8]}$ have shown that in the outer layer the turbulence was consistently suppressed along the ramp, while in the inner layer, it was suppressed only in a small region around the expansion corner and the near-wall quasi-streamwise vortices were well preserved. Generally, the outer layer responds very slowly to a disturbance. The relaxation length becomes larger because the shear stress may take longer than the mean velocity to relax.

Arnette et al. ${ }^{[8,9]}$ used filtered Rayleigh scattering to investigate the expansion effects on a Mach 3 TBL subjected to four expansion regions (centered and gradual expansions of $7^{\circ}$ and $14^{\circ}$ ). The experiments showed that large-scale structures in the outer part of the boundary layer 
maintained their identity and increased in scale across the expansion, suggesting small-scale motions were quenched, resulting in a more intermittent boundary layer. However, they didn't mention how the small scale structures were regenerated in the recovery region. So far the recovery process of supersonic flow over an expansion corner is not fully understood. For a supersonic flow at $\mathrm{Ma}=3$ with $7^{\circ}$ and $14^{\circ}$ expansions, experiments by Arnette et al. ${ }^{[9]}$ and Dawson et al. ${ }^{[10]}$ showed that the boundary layer remains far from fully recovered $19.3 \delta$ downstream of the expansion corner ( $\delta$ is the incoming boundary layer thickness). Measurements of Dussauge and Gaviglio ${ }^{[4]}$ even showed that the incoming boundary layer re-laminarized downstream and the subsequent evolution of the outer part of the boundary layer is very slow. Chung et al. ${ }^{[1]}$ performed experiments for supersonic flow past expansion corners at $\mathrm{Ma}=8$ and 1.28 with small deflection angles, focusing on the surface pressure fluctuations, which showed that the boundary layer did not complete the recovery process. Until now experiments have not been able to provide a complete picture of the recovery process, including the statistics and three-dimensional evolution of turbulent structures.

Another important issue of supersonic boundary layer over expansion corners is its ability to resist flow separation. Zheltovodov et al. ${ }^{[12]}$ concluded that the increment in the 'fullness' of the boundary layer velocity profile after the expansion fan can increase its resistance to separation compared to an isolated compression corner case. Recently experiments were performed for an $\mathrm{Ma}=3.46$ supersonic combustor with a $3.5^{\circ}$ expansion wall connected to a flat isolator ${ }^{[13]}$. Results showed that the separation region on the expansion wall induced by the back pressure is significantly larger than that on the opposite flat wall, which resulted in an asymmetric flame distribution. This phenomenon indicates that the boundary layer along the longer expansion wall may have a weakened ability to resist the adverse pressure gradient. Therefore, the relevance of the velocity profile 'fullness' of the boundary layer in a recovery process to its ability to resist separation is debatable. How to evaluate the resistance of a TBL to flow reversal is still not clear.

Nguyen and Behr ${ }^{[14]}$ showed that Reynolds-Averaged Navier-Stokes approaches gave limited insight into the general flow features in the non-equilibrium region of the expansion flow. More accurate and high-fidelity numerical methods such as direct numerical simulation (DNS) and large-eddy simulation (LES) would be necessary. Many DNS databases of wall-bounded supersonic flows are attainable, including fundamental supersonic boundary layer ${ }^{[15-18]}$, supersonic boundary layer interaction with shock waves ${ }^{[19,20]}$, and supersonic boundary layer over a 
compression ramp ${ }^{[21]}$ etc. Recently Fang et al. ${ }^{[22]}$ calculated a supersonic flow at $\mathrm{Ma}=2.9$ over an expansion-compression corner and found that the re-laminarized expansion flow is far from recovered before the separation region in the compression corner.

This paper presents DNS studies of supersonic flows at Mach 2.7 over expansion corners with deflection angles of $0^{\circ}$ (i.e. a flat plate), $2^{\circ}$ and $4^{\circ}$ to capture the recovery process. Detailed turbulence structures and flow statistics are analyzed, and a shape factor is introduced to evaluate the fullness of the boundary layer velocity profile. The rest of the paper is organized as follows. In Section 2 the numerical method and the simulation parameters are introduced. Section 3 discusses statistical quantities such as mean profiles, boundary layer thicknesses, and pressure fluctuations. Section 4 discusses turbulent structures of the expansion flow. Statistical quantities such as turbulence intensities, turbulent kinetic energy (TKE) and its budget, shape factor are compared in Section 4. Finally, conclusions are given in Section 5.

\section{COMPUTATIONAL SETUP}

\subsection{Numerical simulation and inflow condition}

All simulations in this paper solve the three-dimensional unsteady compressible NavierStokes equations directly without any modelling, using an in-house code. The code has been previously used for studies of instability, transition and turbulence in high-speed flows ${ }^{[20]}$. Here we provide a brief summary of the main features of the code and explain the numerical setup for the present study. Details of the governing equations and algorithm can be found in Touber and Sandham ${ }^{[23]}$ and the references cited therein.

The governing equations are solved using an entropy-splitting approach for the Euler terms and fourth-order accurate finite differences for spatial discretization. In the present work no subgrid model is used, and simulations were run with fine grids to resolve all turbulence scales. A third order Runge-Kutta scheme is used for marching in time.

Specifying proper time-dependent turbulent inflow boundary conditions is very important for time-accurate turbulence simulations. The digital filter inflow approach of Xie and Castro ${ }^{[24]}$ is used. A detailed description of the optimized digital filter used here can be found in Touber ${ }^{[19]}$. The digital filter method is robust to the choice of length scales, provided the prescribed length scales are at least as large as the integral length scales of the turbulent flow and sufficient downstream distance is provided to allow for turbulence to develop ${ }^{[25]}$. In the present study, the streamwise 
characteristic length scales for the three velocity components $u, v$ and $w$ are set to $0.65 \delta_{i}, 0.35 \delta_{i}$ and $0.35 \delta_{i}$ (where $\delta_{i}$ denotes the $99 \%$ boundary-layer thickness at the inflow), respectively. Mean and root-mean-square (RMS) profiles are created beforehand from the DNS database of Schlatter and Orlu $^{[26]}$ for incompressible flat-plate TBLs.

The inflow parameters (shown in Table 1) are set in accordance with the $\mathrm{Ma}=2.7$ experiments of Sun et al. ${ }^{[27]}$. The bottom wall $99 \%$ boundary-layer thickness at the inflow $\delta_{i}$, which is used for all simulations as the reference length, is estimated to be $\delta_{i}=5.7 \mathrm{~mm}$. The corresponding compressible (including density variations) boundary-layer displacement and momentum

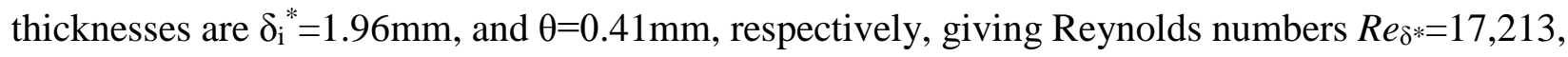
and $R_{\theta}=3,600$ based on inflow quantities. A full list of cases conducted in the present study is given in Table 2 and a sketch of the computational domain is shown in Fig. 1. A curvilinear coordinate system is used, with $x$ being the streamwise direction following the wall, $y$ the direction normal to the local wall and $z$ is the spanwise direction. The coordinate origin is at the expansion corner. Viscosity is assumed to vary with temperature according to Sutherland's law with a reference temperature set as $122.1 \mathrm{~K}$ to match the inflow condition and the Sutherland constant set to $110.4 \mathrm{~K}$.

\begin{tabular}{|c|c|c|c|c|c|}
\hline $\begin{array}{l}\text { Mach } \\
\text { number }\end{array}$ & $\begin{array}{l}\text { Stagnation } \\
\text { temperature }\end{array}$ & $\begin{array}{l}\text { Stagnation } \\
\text { pressure }\end{array}$ & $\begin{array}{l}\text { BL } \quad 99 \% \\
\text { thickness }\end{array}$ & $\begin{array}{l}\text { Momentum } \\
\text { thickness }\end{array}$ & $\begin{array}{l}\text { Reynolds } \\
\text { number }\end{array}$ \\
\hline $\mathrm{M}_{\infty}$ & $\mathrm{T}_{0}$ & $\mathrm{P}_{0}$ & $\delta_{\mathrm{i}}$ & $\theta$ & $\operatorname{Re}_{\theta}$ \\
\hline 2.7 & $300 \mathrm{~K}$ & $100 \mathrm{kPa}$ & $5.7 \mathrm{~mm}$ & $0.41 \mathrm{~mm}$ & 3,600 \\
\hline
\end{tabular}

\subsection{Domain and grid distribution}

Although the digital filter inflow generation can significantly reduce the length required for boundary-layer development, there should nevertheless be enough distance to allow the boundary layers to adjust to an equilibrium state before the expansion corner ${ }^{[24]}$. It was found that a distance of 12 times the inflow boundary-layer $99 \%$ thickness is enough to obtain realistic turbulent mean and RMS profiles from the digital filter inflow generator used ${ }^{[25]}$. Accordingly, the upstream developing length is set to be $L_{i} \geq 12 \delta_{i}$. Here we choose $L_{i}=15 \delta_{i} \approx 85 \mathrm{~mm}$. With the digital filter setup given earlier, the boundary layer thickness at the point of the corner, denoted as $\delta_{0}$, is estimated as 
$\delta_{0} \approx 6.19 \mathrm{~mm}$. As Bradshaw ${ }^{[28]}$ suggested, the recovery length could be ten times the incoming boundary layer thickness. Therefore the expansion length $L_{e}$ is set to be $L_{e} \geq 10 \delta_{0}$. The experiments of Dawson et al. ${ }^{[10]}$ and Arnette et al. ${ }^{[9]}$ for $7^{\circ}$ and $14^{\circ}$ expansion corners at $\mathrm{Ma}=3$ indicated that the boundary layer remains far from recovery even though the downstream length is 20 times the boundary layer thickness. In this study, to simulate the full recovery process, we only consider two small expansion deflection angles at $2^{\circ}$ and $4^{\circ}$ and set $L_{e}=16 \delta_{0} \approx 100 \mathrm{~mm}$. From now on, we denote $2^{\circ}$ expansion case and $4^{\circ}$ expansion case as Expan 2 and Expan 4 , respectively.

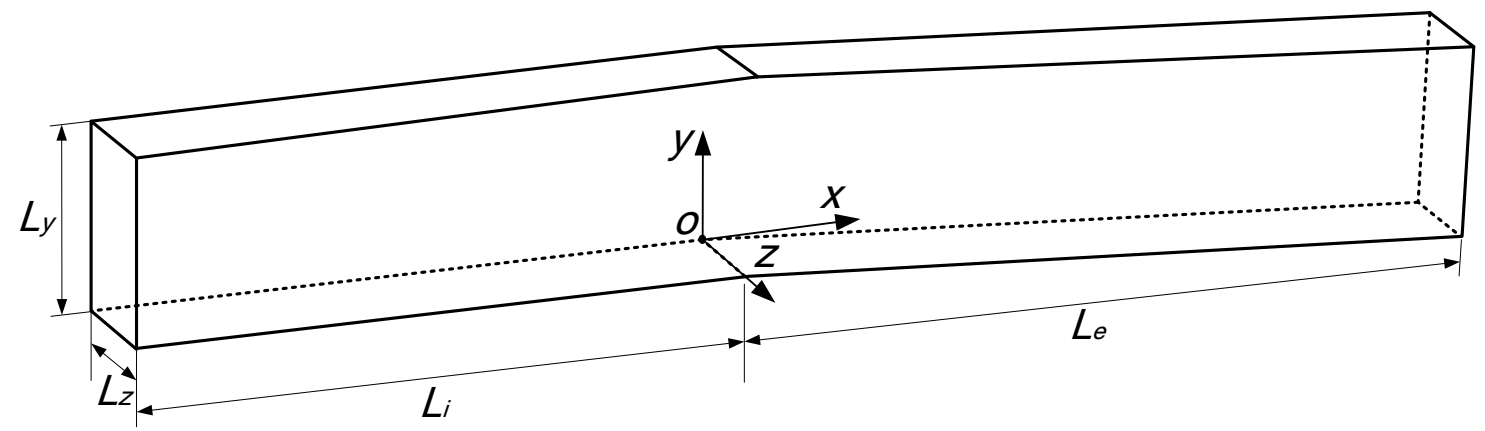

FIG 1. Sketch of the domain used for the simulations.

\begin{tabular}{lllll}
\hline Case & $\begin{array}{l}\text { Domain size } \\
L_{x} \times L_{y} \times L_{z} \\
(\mathrm{~mm})\end{array}$ & $\begin{array}{l}\text { Grid number } \\
N_{x} \times N_{y} \times N_{z}\end{array}$ & $\begin{array}{l}\text { Grid control } \\
y_{b}(\mathrm{~mm}), N_{b}\end{array}$ & $\begin{array}{l}\text { Grid spacing } \\
\beta\end{array}$ \\
& & & & \\
Flat plate/Expan2/Expan4 & $185 \times 26 \times 15$ & $2305 \times 241 \times 289$ & $1.85,88,2.29$ & $6.50 .8-9.84 .1$
\end{tabular}

TABLE 2. Grid numbers and stretching control parameters for different cases

The same number of grid points are used for all cases. The meshes for all cases are the same upstream and downstream, blended smoothly around the expansion corner. A stretched grid is used near the wall in the wall-normal $(y)$ directions, which changes gradually to a uniform grid in the core flow with the required spacing. Grid smoothness is important, especially when high-order schemes are used. Two grid stretching functions with at least second-order continuity at the interface are combined to give coordinates $y_{i}$ in the direction normal to the bottom wall as

$$
y_{i}= \begin{cases}y_{b} \frac{\sinh \left(\beta \eta_{j}\right)}{\sinh (\beta)}, \quad \eta_{j}=\frac{j-1}{N_{b}-1}, & j \leq N_{b} \\ a \cdot j^{5}+b \cdot j^{3}+c \cdot j+y_{\text {mid }}, & j>N_{b}\end{cases}
$$

where $y_{\text {mid }}$ is the middle of the domain, while the parameters $a, b$ and $c$ are fixed by continuity up 
to second order. A predefined distance from the wall, $y_{b}$, is used to specify the region for grid stretching, with $N_{b}$ the number of grid points to be placed inside this region. The fifth-order polynomial enables the grid to change quickly from a highly stretched grid near the wall to an almost uniform grid away from the wall.

In the $y$ direction, the minimum grid spacing is $y_{1}^{+}=0.8$ for the first point off the wall and the maximum is $\Delta y_{\max }^{+}=9.8$. The streamwise direction has a uniform grid distribution, with $\Delta x^{+}=6.5$ based on the inflow TBL quantities. A uniform grid is used in the spanwise direction with a fixed $\Delta z^{+}=4.1$. A grid sensitivity study was performed by coarsening the grid in all directions. The most sensitive feature was found to be the peak skin friction in the vicinity of the expansion corner which varied by less than $5.6 \%$ for a grid that was coarsened by $50 \%$ in all directions.

No-slip boundary conditions were enforced on the bottom wall (shown in Fig. 1). The wall temperature is fixed to the stagnation temperature of the inflow. An integrated characteristic boundary condition is applied to the top boundary. The characteristic boundary condition is applied at the outflow boundary. Periodic boundary conditions are applied in the spanwise direction.

\section{Turbulent statistics on expansion ramps}

\subsection{Comparison of mean profiles with DNS results of Schlatter \& Orlu ${ }^{[26]}$}

Statistics discussed below are based on averaging flowfields over 400 non-dimensional time units. In the simulations the mean inflow profiles were generated from similarity solutions using the same approach as used by Touber and Sandham ${ }^{[23]}$, while the DNS results of Schlatter and $\mathrm{Orlu}^{[26]}$ for a similar Reynolds number are used to provide the inflow RMS values by using Van Driest transformation. Turbulent boundary-layer mean profiles and distributions of RMS values for the flat plate case at $x=0$, which is at a distance of $85 \mathrm{~mm}$ from the inlet, are first evaluated and validated against existing results.

The boundary layer thickness at $x=0 \mathrm{~mm}$ is $\delta_{0} \approx 6.19 \mathrm{~mm}$ and the compressible boundary-layer displacement and momentum thicknesses are $\delta_{0}{ }^{*}=2.37 \mathrm{~mm}$ and $\theta_{0}=0.48 \mathrm{~mm}$, respectively. The Reynolds number based on the momentum thickness $\operatorname{Re}_{\theta}=\rho_{e} U_{e} \theta_{e} / \mu_{w}$ is 4,215. A compressible scaling by the mean density ratio, as suggested by Morkovin ${ }^{[29]}$ and validated by Duan et al. ${ }^{[30]}$ for high Mach numbers, was used to transform current compressible RMS results to compare with incompressible values of Schlatter and Orlu ${ }^{[26]}$. The boundary-layer displacement and momentum 
thicknesses are transformed to $\delta_{0}{ }^{*}=2.50 \mathrm{~mm}$ and $\theta_{0}=0.55 \mathrm{~mm}$, respectively. The corresponding Reynolds number under the van Driest scaling, i.e. $\operatorname{Re}_{\theta, v d}=\rho_{w} U_{e}^{v d} \theta^{v d} / \mu_{w}$, is 1,078 , which is very close to the Reynolds number of 1,000 in the incompressible case of Schlatter and Orlu ${ }^{[26]}$. The subscripts ' $e$ ' and ' $w$ ' above denote the main flow and inner parameters parameter, while the superscript ' $v d$ ' means the value under the van Driest transform. In a zero pressure gradient, the van Driest transformation is expected to reproduce approximately the log-law ${ }^{[31]}$ of the wall as in incompressible TBL, as shown in Fig. 2 and compared with Schlatter and Orlu ${ }^{[26]}$ with good agreement in the near wall and log regions. The calculated non-dimensional freestream velocity is seen to be higher. Morkovin ${ }^{[29]}$ predicted that scaling by the square root of the mean density profile should collapse RMS data for the streamwise velocity component and possibly the spanwise and wall-normal components. The RMS values collapse very well with the DNS results of Schlatter and $\mathrm{Orlu}^{[26]}$, which indicates that the digital filter inflow generation method used here is effective.

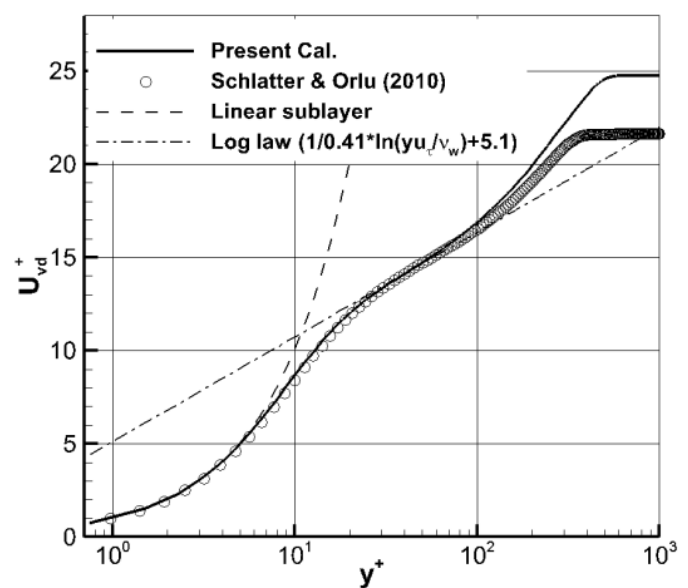

a) Mean velocity distributions

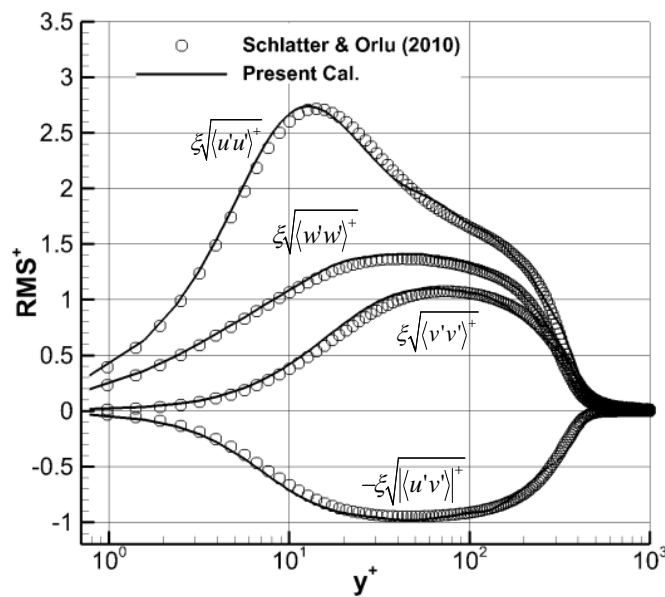

b) RMS velocity distributions

FIG 2. Mean and RMS velocity distributions obtained $85 \mathrm{~mm}$ downstream of the inflow boundary of the flat plate. The van Driest transformation was applied to the mean velocity and a Morkovin scaling $\xi=\sqrt{\rho / \rho_{w}}$ to the RMS curves.

\subsection{Mean properties in the recovery region}

The time-averaged skin friction along the wall is shown in Fig.3. For an incompressible flow, if we assume the mean velocity profile can be represented by $u / U_{e}=(y / \delta)^{1 / 7}$ (where $u$ denotes the local streamwise velocity and $U_{\mathrm{e}}$ the freestream velocity of the boundary layer), the skin friction considering compressibility effects can be calculated following Young's assumption ${ }^{[32]}$, as $c_{f} / c_{f i} \approx\left(1+0.325(\gamma-1) M_{\infty}^{2} \sigma^{1 / 3}\right)^{-0.622}$ for an adiabatic wall, where $c_{f}$ denotes the skin friction 
coefficient for compressible flow, $c_{f i}$ the skin friction coefficient at the same Reynolds number in incompressible flow and $\sigma$ is the Prandtl number. If we use the transformed boundary layer thickness to estimate the $\operatorname{Re}_{x}$ of the boundary layer by $\delta^{v d}=0.37 x \operatorname{Re}_{x}^{-0.2}$ and $c_{f i}$ is set as $c_{f i}=0.0592 \mathrm{Re}_{x}^{-0.2}$, we get $c_{f} \approx 0.00194$ with $\sigma=0.72$ or $c_{f} \approx 0.00189$ with $\sigma=0.9$ at the expansion point $\left(x / \delta_{0}=0\right)$, which is $85 \mathrm{~mm}$ downstream of the inlet. This estimation is comparable to the numerical calculation $c_{f} \approx 0.00171$. The skin friction coefficient for different cases drops and adapts to the flat plate TBL results from $x / \delta_{0}=-5$ onwards, which indicates that the artificial inflow specified by the digital filter develops to a fully TBL before the corner. Therefore the upstream boundary layer development distance of $14 \delta_{0}$ used is appropriate for the specified inflow to develop to an equilibrium state. The sudden wall pressure drop and skin friction rise downstream of the corner (located at $x / \delta_{0}=0$ ) seen in the expansion cases are due to the expansion wave. The skin friction coefficient around the corner is very high. The wall pressure decreases and recovers to the flat plate value more slowly than the skin friction, which indicates that the skin friction is more related to the flow physics in the inner part of the boundary layer, while the wall pressure depends on events across the whole boundary layer. The pressure gradient and a renormalized pressure gradient $(d P / d x) /\left(\delta^{*} / \tau_{w}\right)$ are shown in Fig. 4c) and $\left.4 \mathrm{~d}\right)$, where $\tau_{w}$ is the wall shear stress and $\delta^{*}$ is the displacement thickness of the boundary layer. It can be seen that the pressure gradient has a negative maximum in the range $0<x / \delta_{0}<5$, which corresponds to the non-equilibrium behaviour discussed above. For $x / \delta_{0}>5$, the pressure gradient approaches zero, however the turbulence retains characteristics from the upstream region $0<x / \delta_{0}<5$ and is far from being in equilibrium. This will be discussed further in section 5.3. 


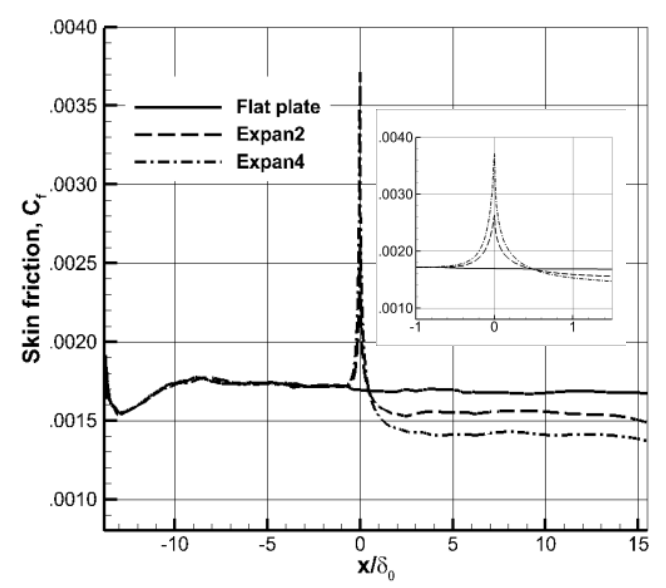

a) Skin friction coefficient

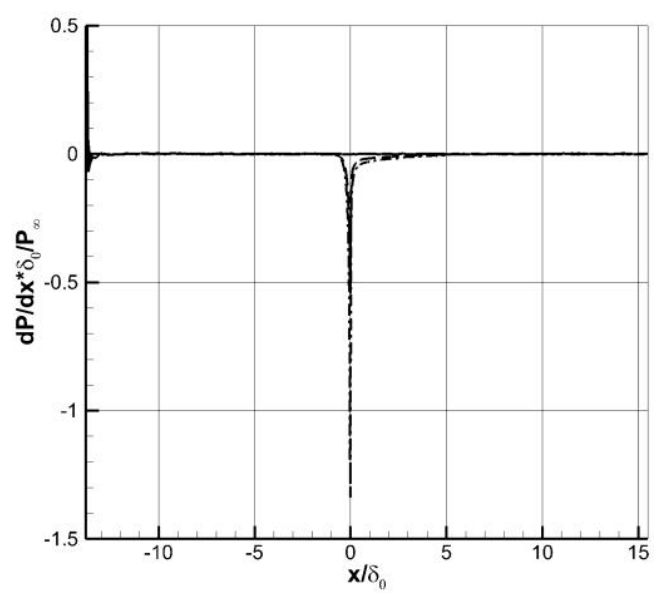

c) Pressure gradient

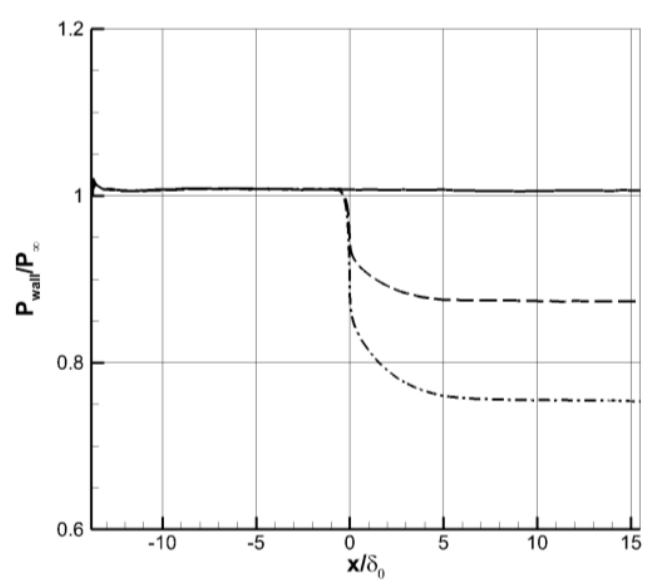

b) Wall pressure

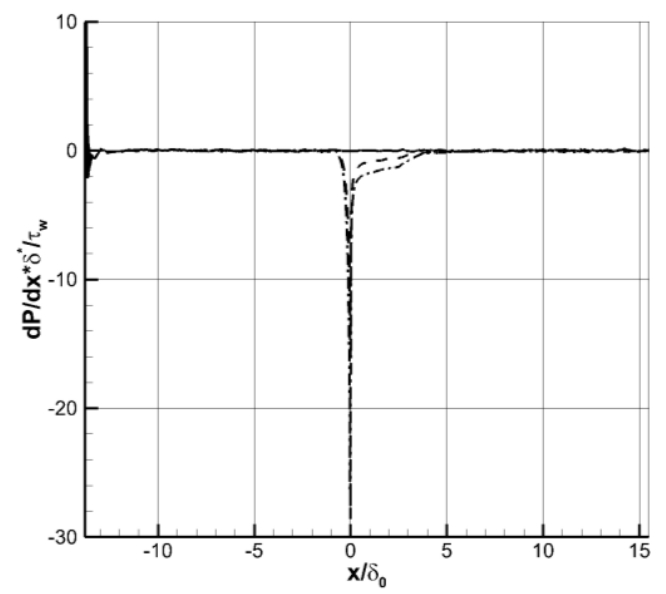

d) Non-dimensional pressure gradient

FIG.3 Skin friction coefficient and wall pressure distributions: a) skin friction coefficient, b) wall pressure normalized by the freestream static pressure, c) pressure gradient and d) non-dimensional pressure gradient.

Mean velocity profiles are compared for the flat plate and expansion cases in Fig.4 at several streamwise locations both upstream and downstream of the expansion corner. The location selected just downstream of the corner (at $x / \delta_{0}=0.81$ ) is within the strong interaction area underneath the expansion fan. The expansion cases have higher velocity over most of the boundary layer compared to the flat plate in the vicinity downstream of the corner (for brevity not given in Fig.4). To clarify the profile fullness Fig. 4 give the velocity non-dimensionalized by the freestream velocity over the boundary layer $\left(U_{e}\right)$. It can be seen from Fig.4 that the downstream velocity profiles become fuller at $\mathrm{x} / / \delta_{0}=0.81$ as the expansion angle increases. While at $\mathrm{x} / \delta_{0}=5.65$, and $x / \delta_{0}=15.35$ it is not easy to tell the fullness directly since the velocity profile in the outer layer obviously gets changed. In Fig.5, the velocity profiles are plotted in wall coordinates using the van Driest transformation ${ }^{[33]}$ which takes into account the variation in density for compressible boundary layers. It is found that after 
the expansion corner the velocity profiles depart significantly from the log-law and the mean velocity overshoots the log law in the buffer layer (Fig. 5b). At $x / \delta_{0}=5.65$ (Fig. 5c) the velocity profile follows the law of the wall up to $y^{+} \approx 20$ for the expansion cases. Further downstream, the sub-layer returns to the shape normally seen in a fully-developed TBL with zero pressure gradient and the log layer reappears (Fig. 5d). A careful observation of the velocity profiles finds that the velocity decreases in the inner layer of the expansion cases compared to the flat plate. The mean velocity profile at $x / \delta_{0}=15.35$ is re-plotted using outer scaling in Fig.6 using a logarithmic scale for the $x$ axis. It is clear that although flow is accelerated after the expansion (shown in Fig.6a) the velocity profiles in the inner layer $\left(y / \delta_{0}<0.1\right)$ experience a reduction as the expansion angle increases. This is clearer in Fig.6b) when $u / U_{e}$ is used on y-axis. This result challenges the traditional knowledge of the fullness of velocity profiles of expansion ramps. This will be discussed in more detail in Section VI.

a)
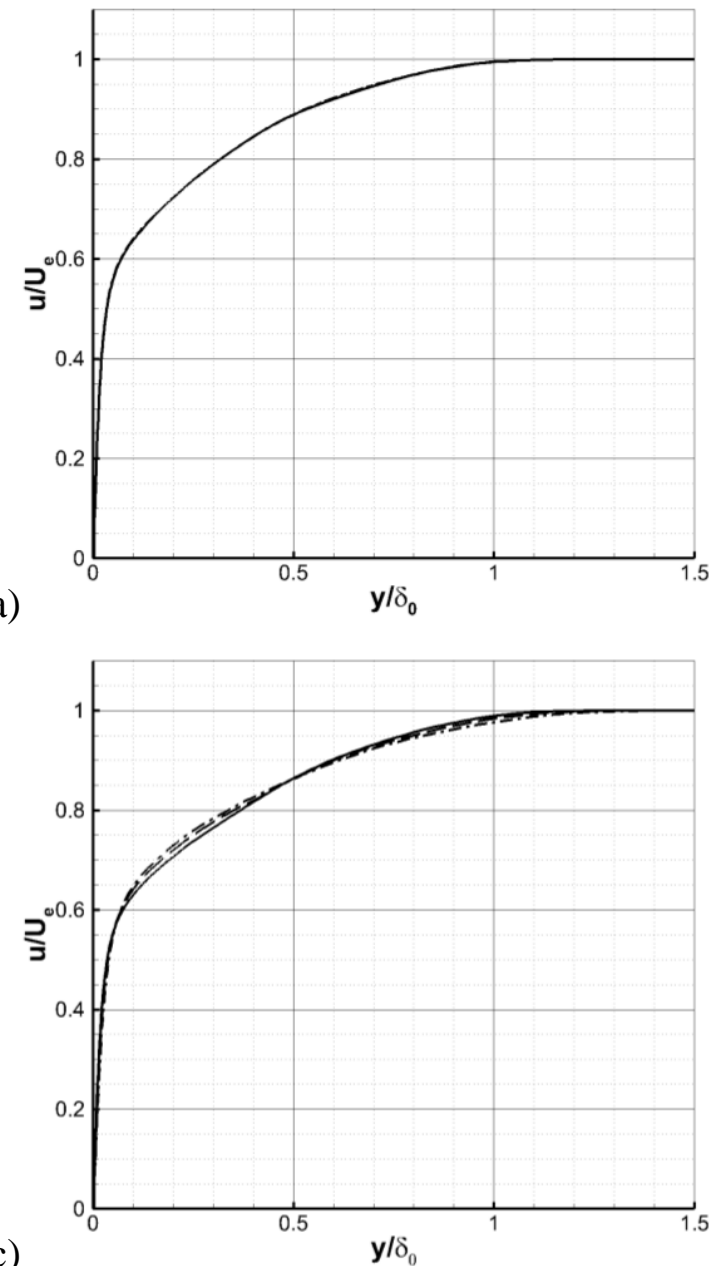

b)

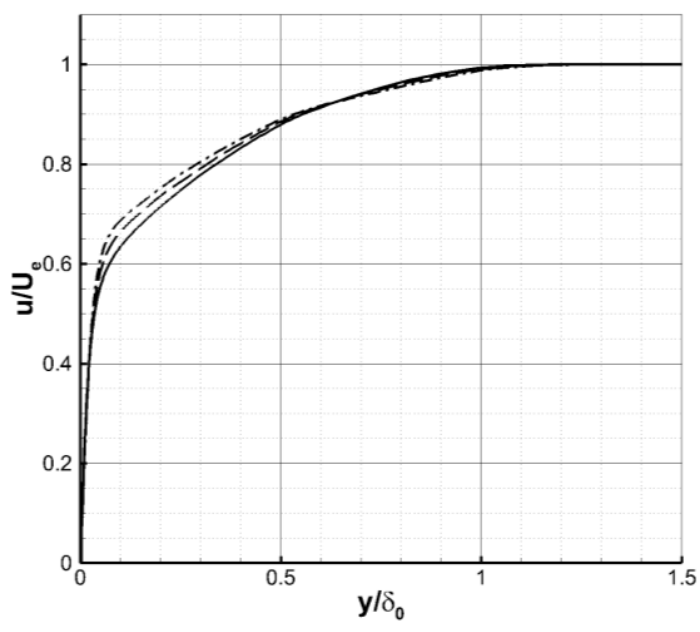

d)

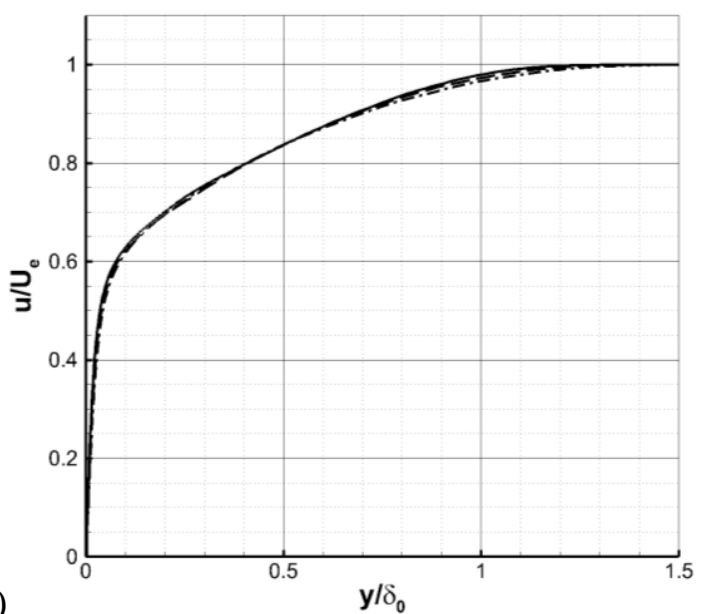

FIG.4 Mean velocity profiles at different locations, a) $x / \delta_{0}=-2.42$, b) $x / \delta_{0}=0.81$, c) $x / \delta_{0}=5.65$, d) $x / \delta_{0}=15.35$, normalized by local freestream velocity over the boundary layer $\left(U_{e}\right)$. Line legend is same with Fig.3a). 

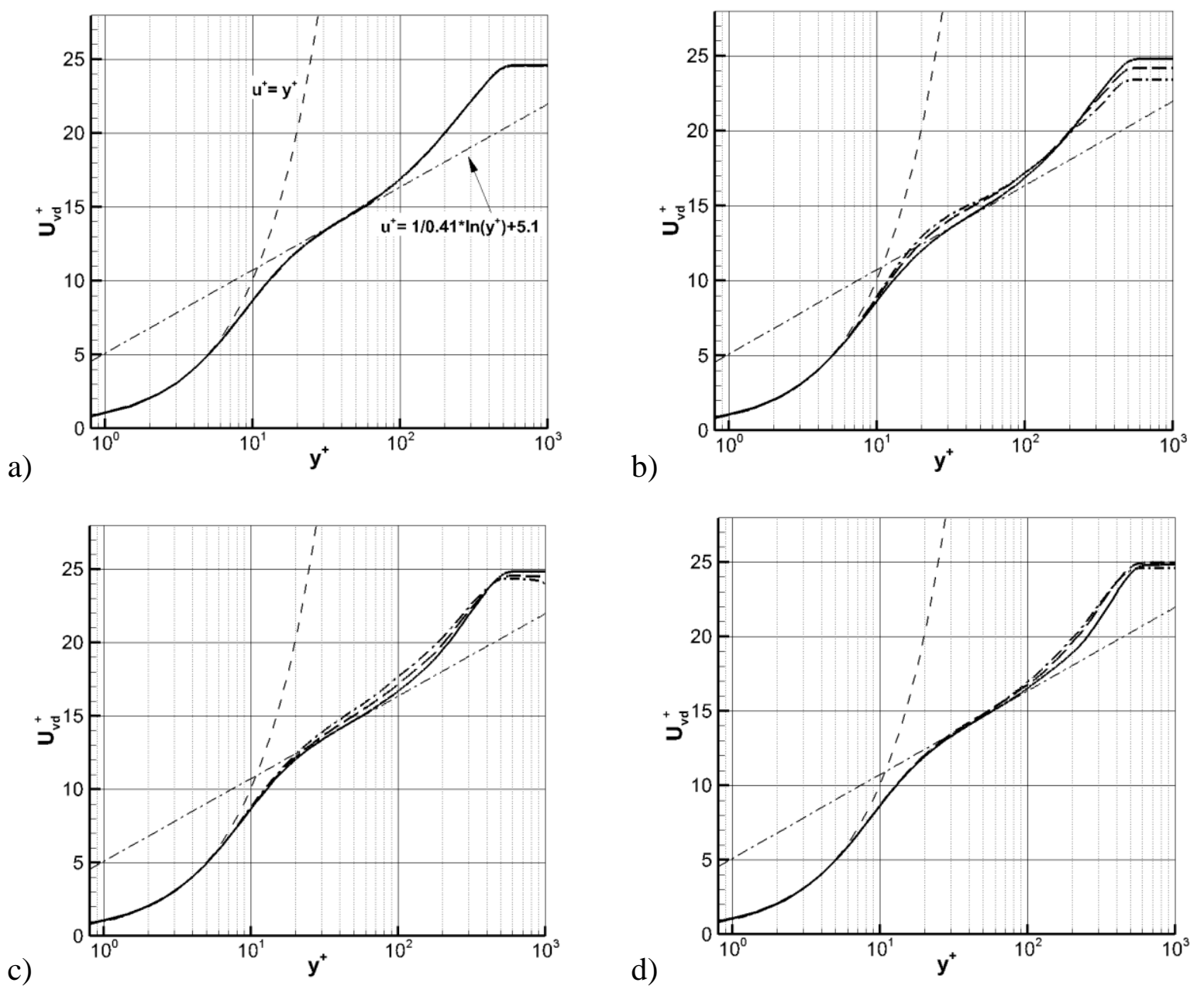

FIG.5 Mean velocity profiles at different locations, with van Driest transformation applied, a) $x / \delta_{0}=-2.42$, b) $x / \delta_{0}=0.81$, c) $x / \delta_{0}=5.65$, d) $x / \delta_{0}=15.35$. Line legend is same with Fig.3a).

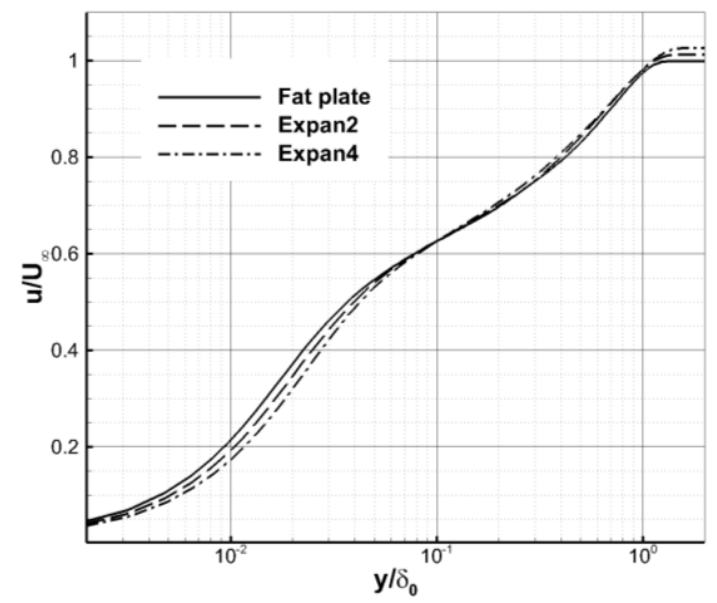

a) Normalized by $U_{\infty}$, the inflow freestream velocity

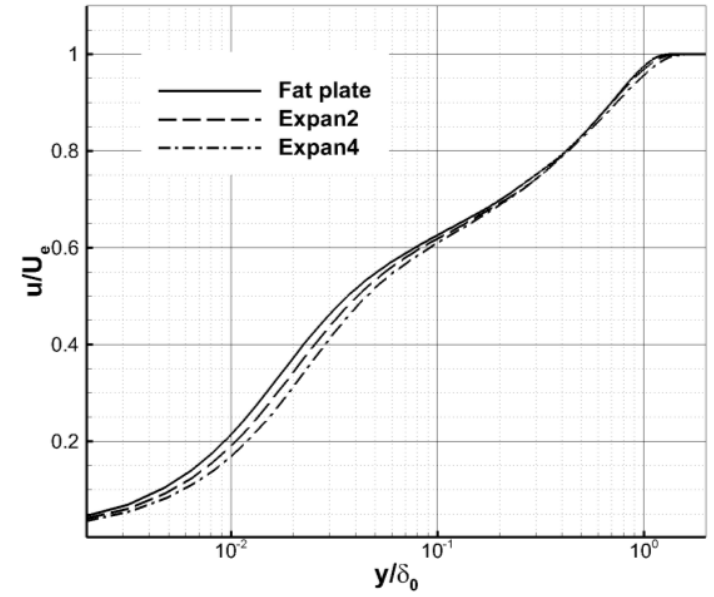

b) Normalized by $U_{e}$, the local freestream velocity

FIG.6 Distribution of velocity as a function of wall-normal distance at $x / \delta_{0}=15.35$.

The displacement thickness $\delta^{*}$ and the momentum thickness $\theta$ are defined as 


$$
\begin{aligned}
& \delta^{*}=\int_{0}^{\infty}\left(1-\frac{\rho}{\rho_{e}} \frac{u}{U_{e}}\right) d y \\
& \theta=\int_{0}^{\infty} \frac{\rho}{\rho_{e}} \frac{u}{U_{e}}\left(1-\frac{u}{U_{e}}\right) d y
\end{aligned}
$$

Fig.7 gives the calculated displacement and momentum thicknesses along the streamwise direction for different cases. The displacement thickness $\delta^{*}$ experiences a rapid rise across the corner, while the momentum thickness $\theta$ experiences a rapid drop downstream of the corner before recovering to the flat plate values further downstream. The boundary layer thicknesses after recovery are seen to be only slightly increased due to the expansion effect. For expansion cases, the recovery is complete before the outlet. A slightly earlier recovery is observed for Expan2 than for Expan4. Therefore the remaining part of the paper will concentrate on analyzing data from the Expan4 case to get a more complete understanding of the recovery process.

Previous studies ${ }^{[1,34]}$ concluded that a fuller streamwise velocity profile increases the ability of a boundary layer to resist flow separation. For a supersonic expansion flow, as we discussed above, although the increase in the velocity leads to a fuller velocity profile in the outer part of the boundary layer, the velocity in the inner part of the boundary layer for the expansion cases decreases, which results in a thicker boundary layer compared with the flat plate, as shown in Fig. 7. It is difficult to quantify the fullness of the boundary layer after equilibrium regeneration on the expansion ramp. In order to evaluate the boundary layer, including the resistance to an adverse pressure gradient, shape factor is often used as an indicator. Usually an increase in the shape factor of a supersonic boundary layer indicates that flow separation is more likely to occur on the wall.
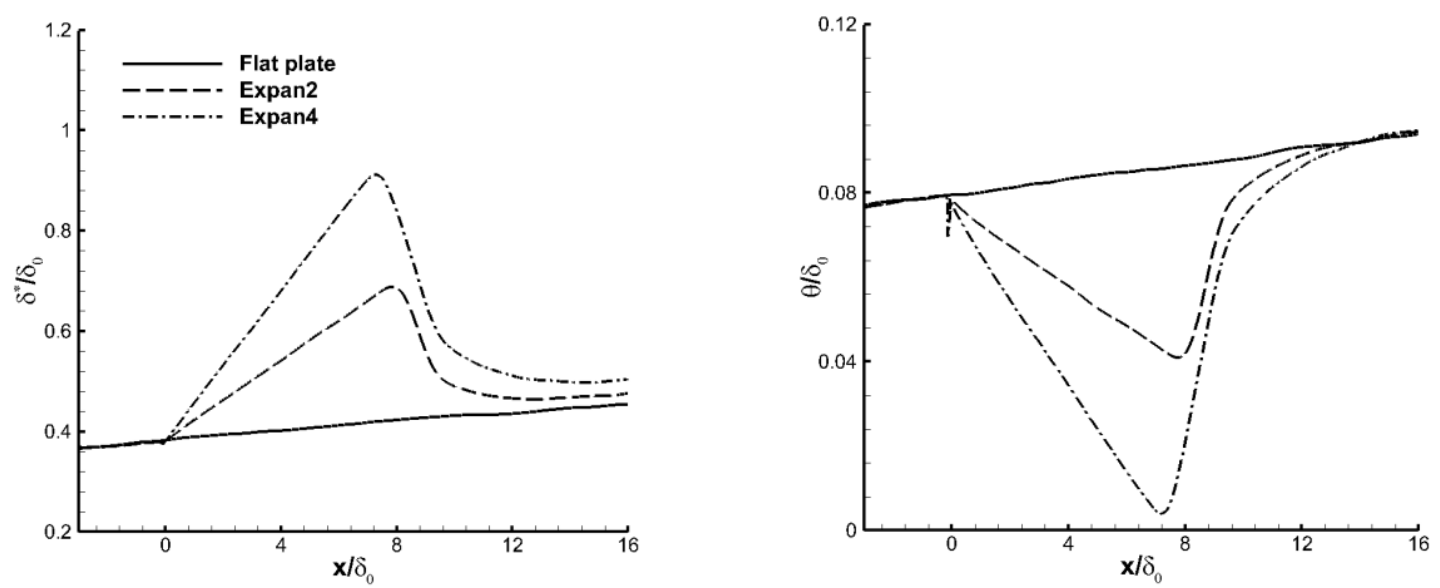

FIG.7 Development of the displacement $\left(\delta^{*}\right.$, left $)$ and momentum $(\theta$, right) thicknesses along the streamwise direction. 
The shape factor $H=\delta^{*} / \theta$, defined as the ratio of displacement thickness $\delta^{*}$ to momentum thickness $\theta$, is calculated based on boundary layer thickness results shown in Fig.7 and is shown in Fig. 8. We will first evaluate the validity of the value shown in Fig.8 from a theoretical viewpoint. For an incompressible boundary layer, assuming a mean velocity profile of $u / U_{e}=(y / \delta)^{1 / n}$, the shape factor is given by $H=\delta^{*} / \theta=(2+n) / n$, which gives $H_{i} \approx 1.286$ for $n=7$. Following Monaghan ${ }^{[35]}$, the shape factor for a supersonic TBL can be related to $H_{i}$, the free stream temperature $T_{\infty}$, the wall temperature $T_{w}$, and the recovery temperature $T_{r}$, by the following equation

$$
\frac{H}{H_{i}}=1+\left(\frac{T_{w}}{T_{\infty}}-1\right)+\left(\frac{T_{r}}{T_{\infty}}-1\right) .
$$

For an adiabatic wall this equation becomes,

$$
\frac{H}{H_{i}}=1+r(\gamma-1) M a_{\infty}^{2}
$$

where $r$ is the recovery coefficient. This equation indicates a large shape factor for supersonic flow at high Mach number, implying that boundary layer displacement effects become much larger than the momentum thickness as Mach number increases. For example, for air $(\gamma=1.4)$ at $\mathrm{Ma}=2.7$ when $r=1.0$, we get $H=5.03$; if $r=0.85, H=4.47$. It is found that the shape factor of the flat plate at $x / \delta_{0}=15.6$ is 4.845 , which just falls in the range of [4.47, 5.03].

In expansion flow, the upstream flow history, outer layer exchange and inner layer turbulence generation can all influence the boundary layer development. A favourable pressure gradient can result in a fuller velocity profile in the outer boundary layer on the ramp, however, the fullness level suffers a decrease in the inner layer since the turbulence decay in the outer layer inhibit the high momentum in the freestream spread into the inner layer. The TKE in the inner layer is high, which represents large momentum dissipation from the inertial flow and leads to a decrease in the mean velocty magnitude. The shape factor is an integration from the inner layer to the outer layer, which could be used as an indictor to evalute the fullness level from an overall aspect.

Fig. 8 shows the shape factor as a function of the streamwise location for all three cases. It is clearly seen that the shape factor increases rapidly after the expansion corner, followed by a sudden decrease in the recovery region and a slow increase in the equilibrium section. Fig. $8 \mathrm{~b}$ ) gives the 
closer view of the shape factor in the equilibrium region. It is found that $H=5.314$ at $x / \delta_{0}=15.6$ for $4^{\circ}$ expansion, $H=5.013$ for $2^{\circ}$ expansion and $H=4.8435$ for flat plate case respectively. The values of shape factor indicate that the fullness level of velocity profile in the flat plate case is still higher than the $2^{\circ}$ and $4^{\circ}$ expansion cases. The shape factor increases as the deflection angle is increased. A higher value of the shape factor implies that the expanded supersonic flow has a lower resistance flow separation. This partially explains why large separation occurred on the expanded wall in previous experimental results ${ }^{[22]}$.

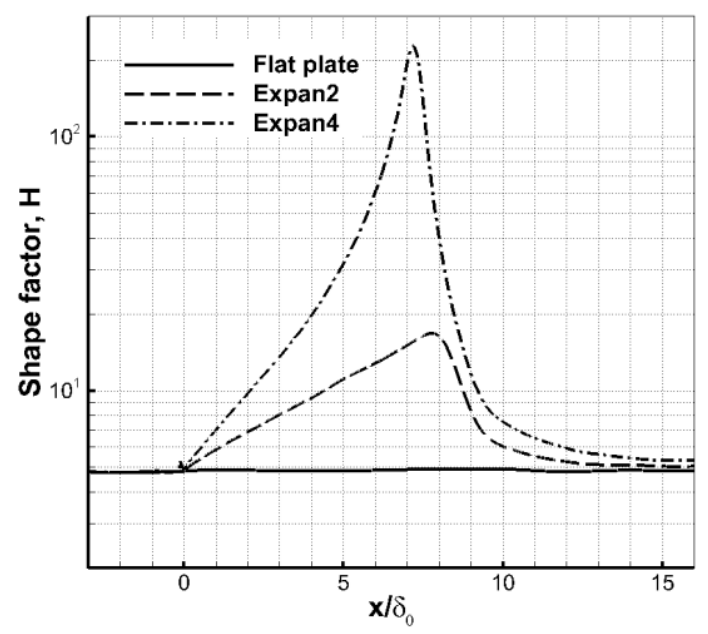

a) shape factor

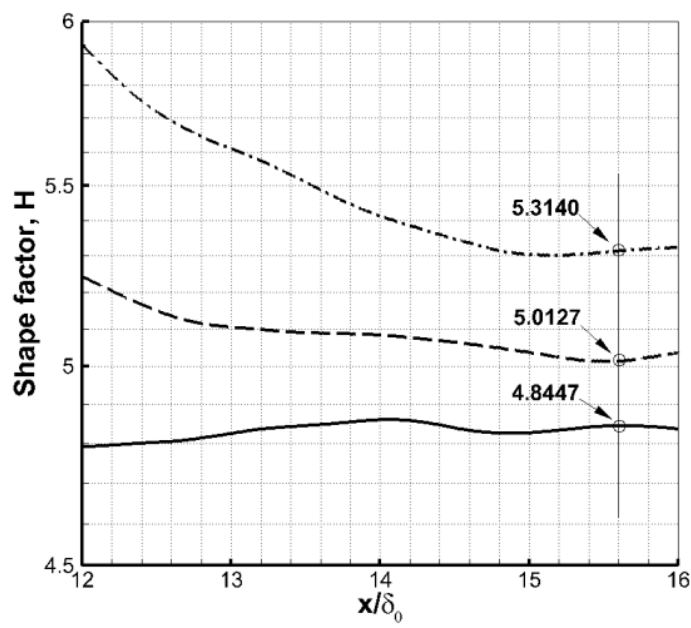

b) closer view of the shape factor near the outlet

FIG.8 Shape factor as a function of streamwise location of different expansion cases.

\section{Instantaneous turbulent structures in the recovery flow}

\subsection{Turbulent structures of density and vortex fields}

Contours on a two-dimensional slice of the instantaneous density field, given in Fig.9, show that the density behind the expansion corner decreases in the main stream and in the boundary layer downstream of the corner. Since the expansion deflection angle is not large, the well-known phenomena of small scale quenching and suppression of the large scale turbulence is not shown clearly in the slices. As can be seen in Fig.9c), supersonic flow passing through an expansion corner with a larger deflection angle is accelerated and the boundary layer becomes thicker, as indicated by the boundary layer properties given in Section 3.2.

In order to investigate the response of vortex structures within the supersonic boundary layer and their changes after the expansion, we considered iso-surfaces of $\lambda_{2}{ }^{[36]}$, which is the second eigenvalue of the $3 \times 3$ matrix comprised of velocity gradient, i.e., $M_{i j}=\sum_{k=1}^{3}\left(\Omega_{i k} \Omega_{k j}+S_{i k} S_{k j}\right)$, where 
$S_{i j}=1 / 2\left(\partial u_{i} / \partial x_{j}+\partial u_{j} / \partial x_{i}\right)$ and $\Omega_{i j}=1 / 2\left(\partial u_{i} / \partial x_{j}-\partial u_{j} / \partial x_{i}\right)$. A small negative value is selected to identify turbulent structures, as shown in Fig.10 for iso-surfaces of $\lambda_{2}$ coloured with the instantaneous temperature from different cases. Comparisons between the flat plate and $4^{\circ}$ expansion cases indicate that turbulence is suppressed for supersonic flow past an expansion corner during the expansion process. Turbulence diminishes rapidly in the $4^{\circ}$ expansion case. The reduction is not homogenous and pockets of high turbulence intensities exist in the flow, which are related to the intermittent turbulent structures coming from upstream of the corner. For example, within the dashed-line on Fig.10c) pockets of diminished structures in the boundary layer can be identified downstream of the corner. A close inspection reveals that the expansion does not attenuate turbulence in the inner part of the boundary layer marked with higher temperature. In the recovery region, the structures in the near wall region are regenerated, these structures develop and mix with the structures in the outer part of the boundary layer.

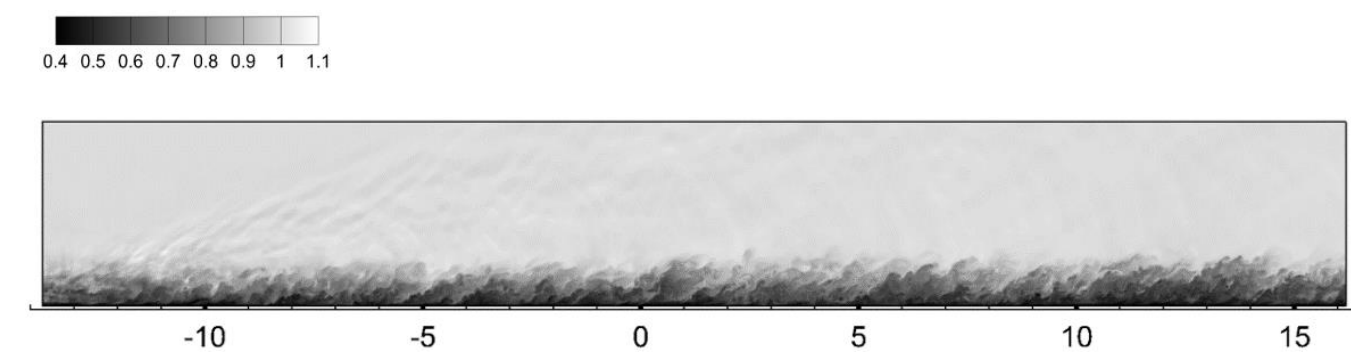

a) flat plate

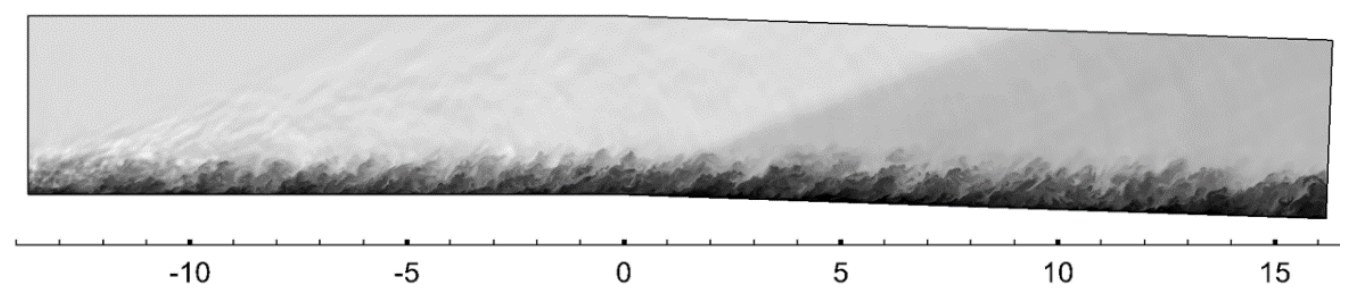

b) Expan2

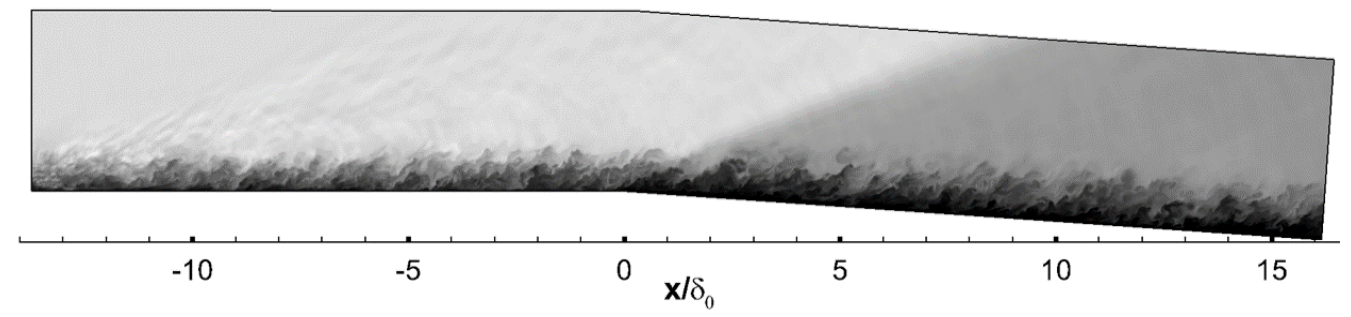

c) Expan4

FIG.9 Density flowfield of the flat plate and the expansion cases, a) flat plate, b) Expan2, c) Expan4, normalized by the inflow freestream density. 

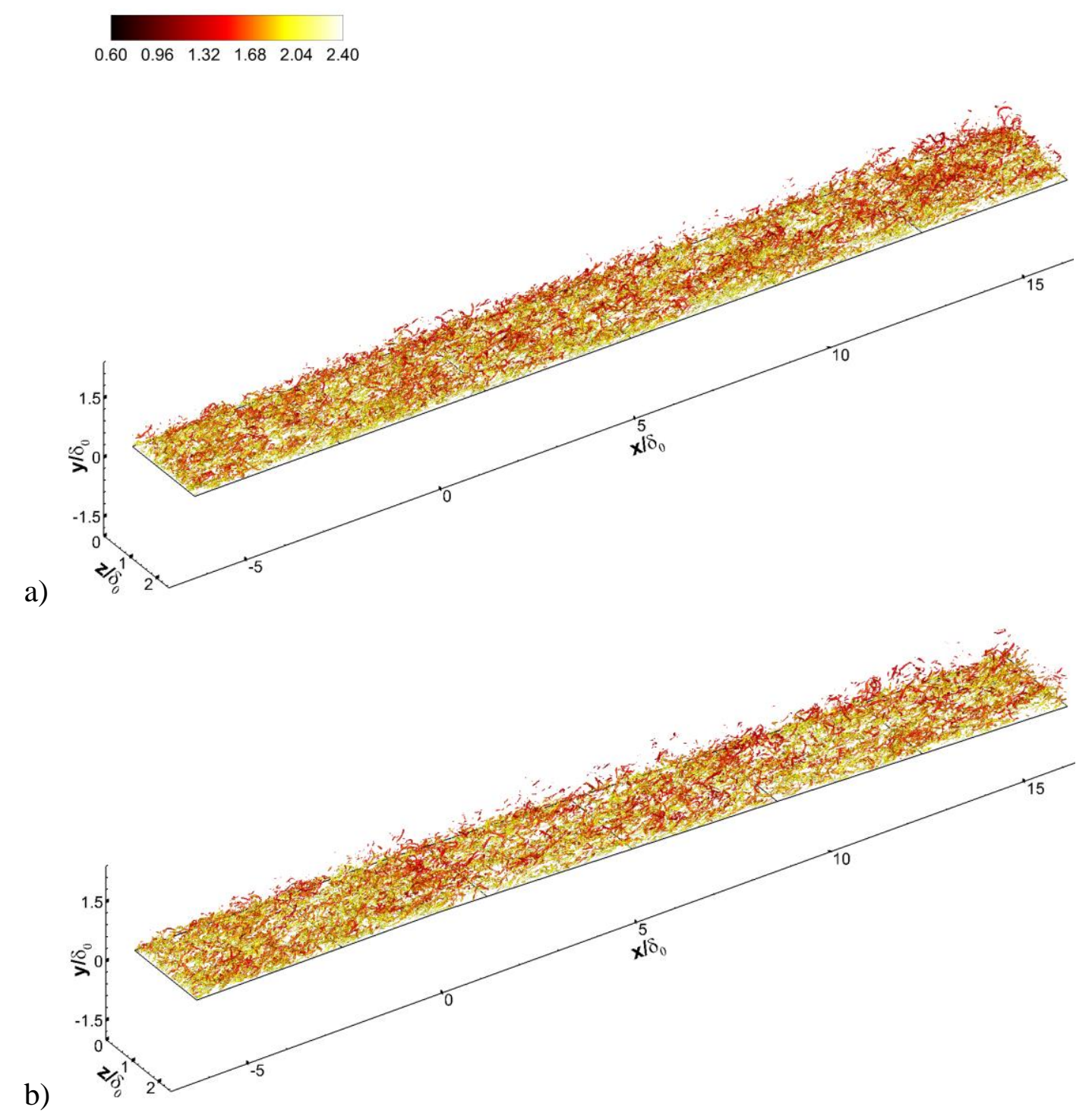

b)

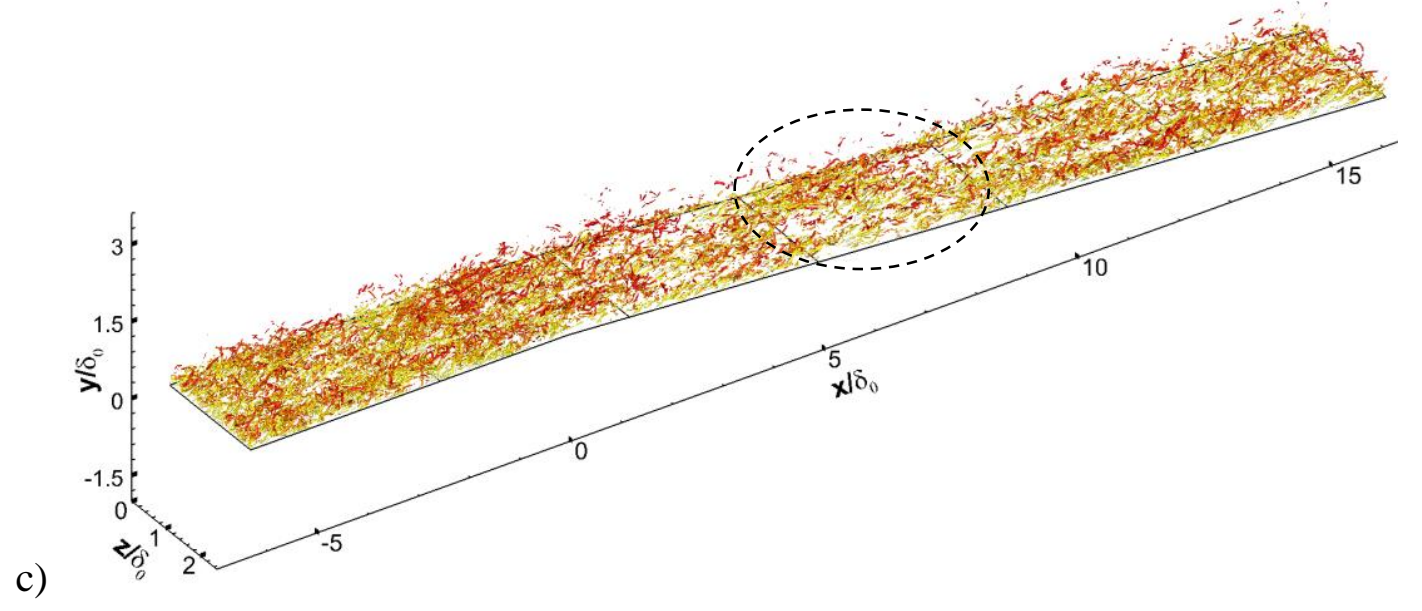

FIG.10 Iso-surface of $\lambda_{2}$ coloured by the instantaneous temperature for different cases: a) flat plate, b) Expan2, c) Expan4. 


\subsection{Comparison of near wall streaks and two-point correlations}

Near wall streaks in the turbulent flowfield from the $4^{\circ}$ expansion case will be analysed to investigate the turbulence decay downstream of the expansion corner. Streamwise velocity fields at a constant distance to the wall are shown in Fig. 11 from a top view for several wall-normal locations, where low speed (dark) and high speed (light) streaks can be clearly identified. The flowfield in the expansion region shows different characteristics to the flat plate. For the $y / \delta_{0}=0.0266$ slice, which is in the near-wall region at $y^{+} \approx 11.5$, the classic streamwise elongated streaks appear in the upstream undisturbed boundary layer region. In the inner layer, however, the quasi-streamwise structures are preserved all the way along the expansion ramp. A careful observation shows that the distance between two neighboring low-speed streaks increases downstream of the expansion corner. This will be analysed quantitatively via two-point correlation later. At $y / \delta_{0}=0.133$, the velocity just downstream of the corner increases suddenly (shown in lighter color) and this phenomenon is accompanied by a reduction in velocity fluctuations is weakened suddenly in the vicinity of the expansion corner. The streamwise velocity contours in the slice show weakened and less organized structures in the expansion boundary layer and these fluctuations are consistently suppressed over the ramp region. A comparison of the streak patterns in the two planes shows that the streaky structures are preserved in the inner boundary layer and weakened in the outer layer. The boundary layer recovers the classic streaky structures further downstream of the expansion corner, which promotes the near-wall turbulence recovery towards the equilibrium state. In the $y / \delta_{0}=0.0266$ slice of the $4^{\circ}$ expansion case, we can see that large-scale streaks, with low speed spots from the turbulence structures in the inner layer, start to appear at $x / \delta_{0} \approx 3$. At $y / \delta_{0}=0.266$, the streaks that appear at large scales in the flat plate case and upstream of the expansion corner, disappear after the expansion, which indicates that the coherent structures from the outer part of the undisturbed boundary layer are damped during the expansion process. The streaks occur again with a lag compared to the $y / \delta_{0}=0.133$ slice. This phenomenon suggests that the turbulence recovery is mainly attributed to the near-wall turbulence growing from the inner layer to the outer layer. 
a)
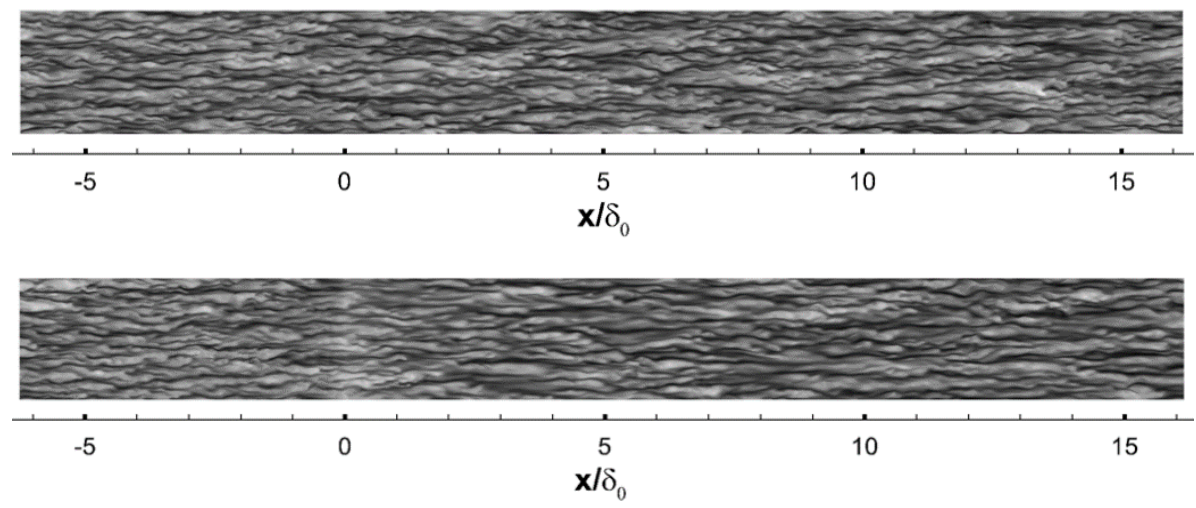

b)
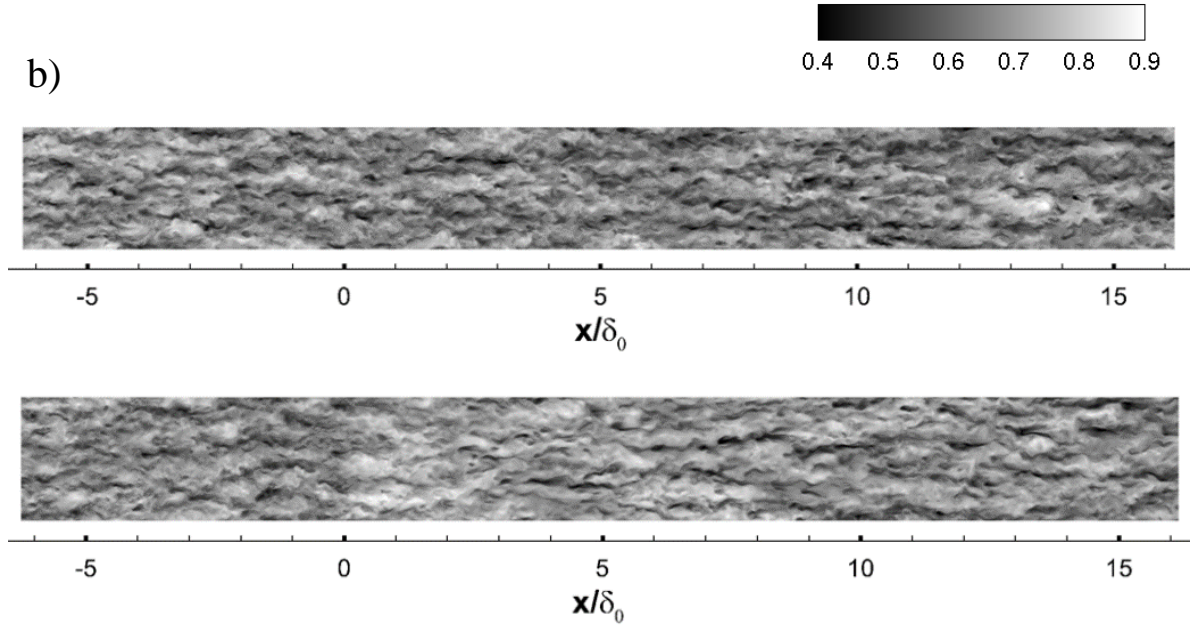

c)
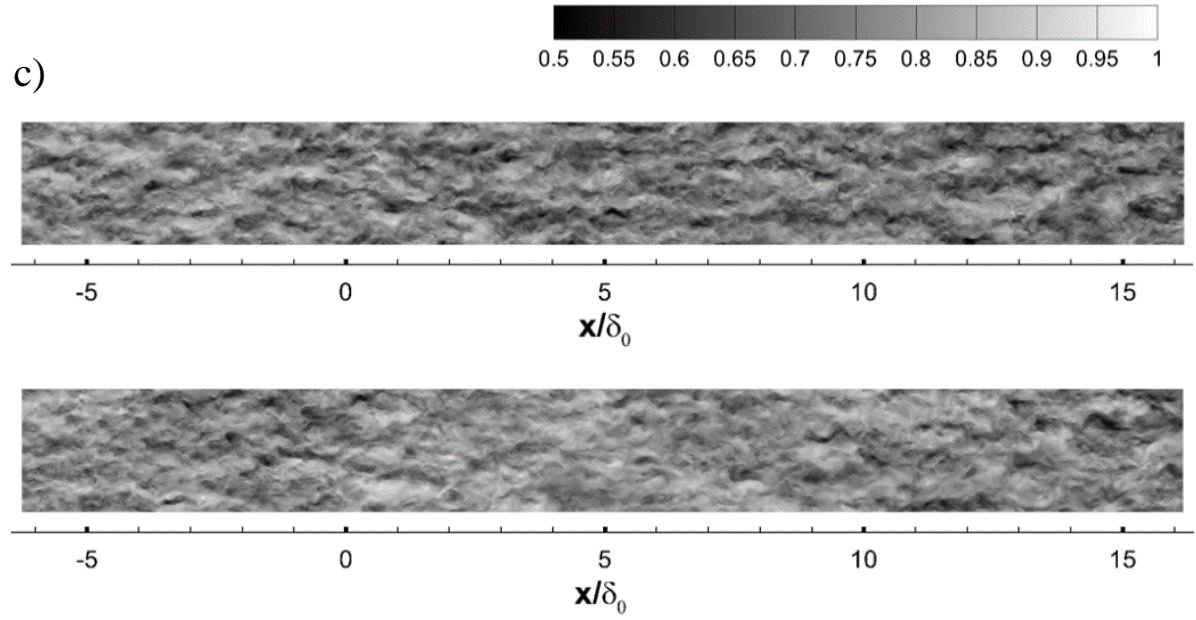

FIG. 11 Streamwise velocity contours of the flat plate (top) and $4^{\circ}$ expansion (bottom) cases, normalized by inflow freestream velocity, for slices at different wall-normal distances: a) $y / \delta_{0}=0.0266$, b) $y / \delta_{0}=0.133$ and c) $y / \delta_{0}=0.266$.

To quantify the distance between neighboring low-speed streaks, two-point correlations of the velocity perturbations are examined for spanwise separations at $x / \delta_{0}=15.5$ at different wallnormal distances (Fig. 12). It can be seen that the wall-normal velocity correlation for the $4^{\circ}$ expansion case always appears at a larger separation compared to the flat plate case. This shows 
that the streak width in the expansion case is larger than on the flat plate. For other wall-normal distances, the increase in the streak width compared to the flat plate case can all be identified.

a)
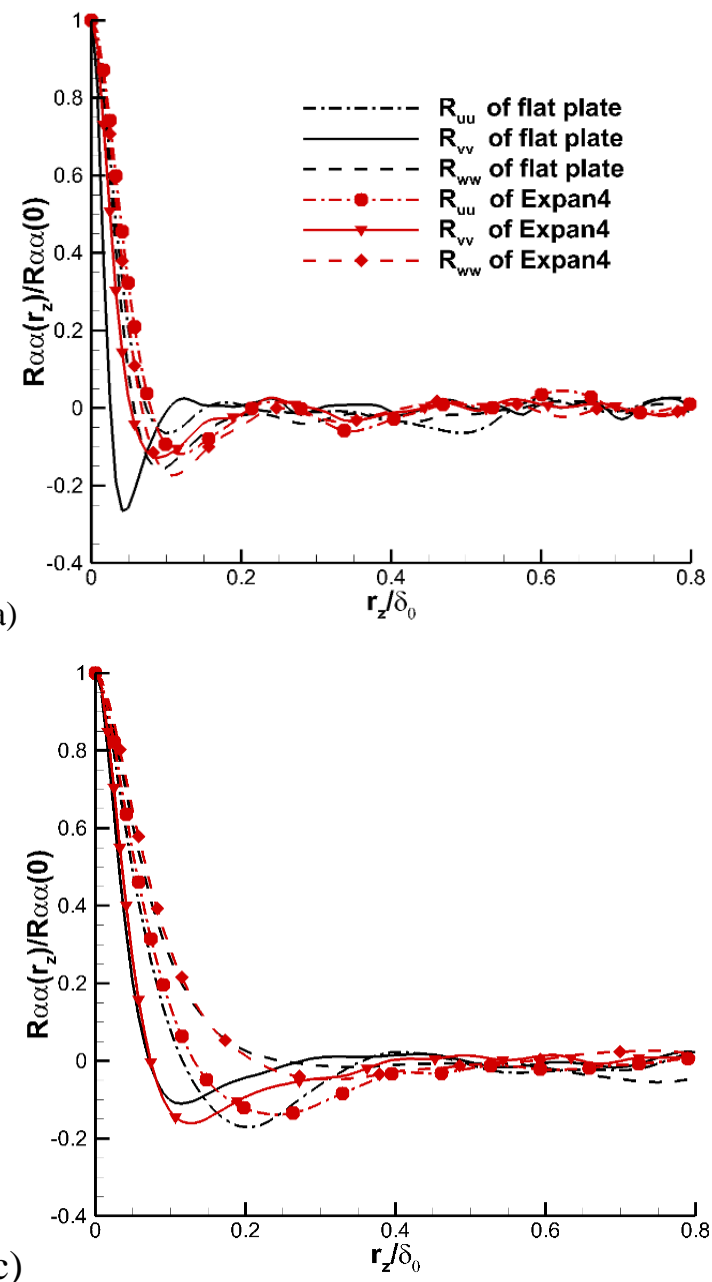

b)

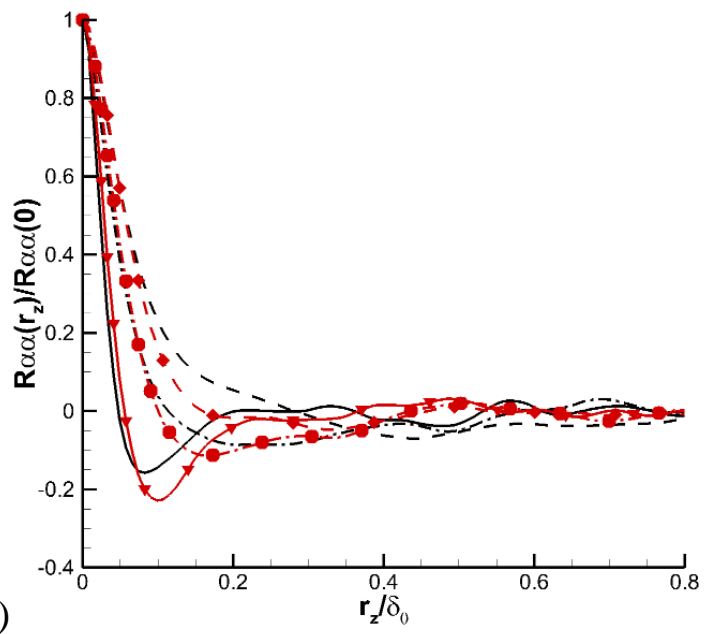

d)

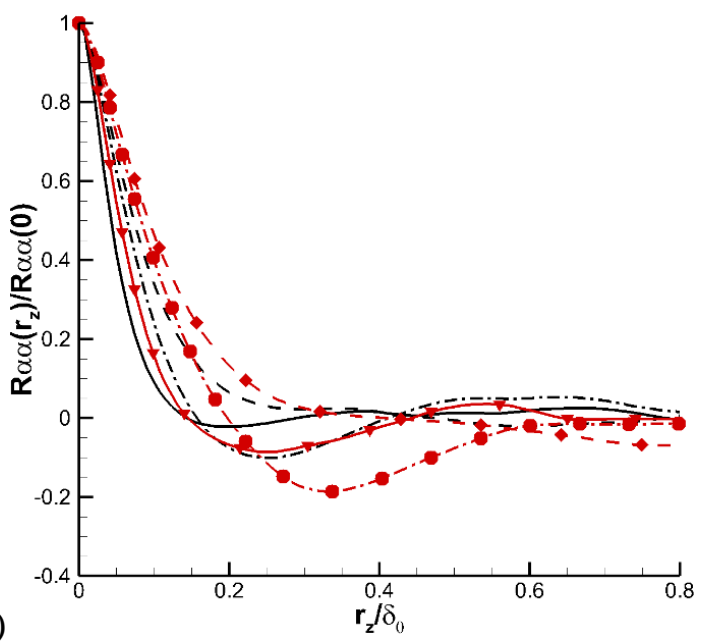

FIG.12 Spanwise two-point correlations for velocity perturbations at different wall-normal distances: a) $y / \delta_{0}=0.0066$,

b) $y / \delta_{0}=0.0562$, c) $y / \delta_{0}=0.1076$, d) $y / \delta_{0}=0.2555$.

\subsection{Iso-surfaces of velocity and temperature in three-dimensional flow}

For easy comparison of the turbulent structures between the flat plate and expansion cases, the expansion domain is transformed to a rectangular domain, as shown in Fig.13, with a transformed velocity $u_{t}=u \cos \beta-v \sin \beta$ and $v_{t}=u \sin \beta+v \cos \beta$, where $\beta$ is the wall deflection angle.

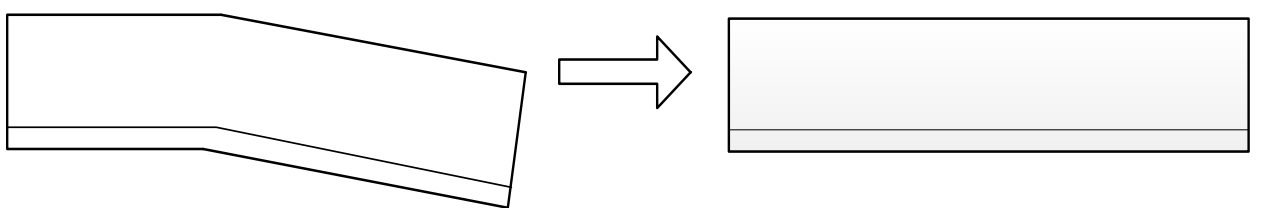

FIG.13 Transformation from an expansion domain to a rectangular domain 
A close-up view of the temperature contours and $u_{t}$ velocity contours is shown in Fig.14, to observe the turbulent strucutures. It is found that large scale structures are preserved in the outer layer downstream of $x / \delta_{0}=0$. To understand the change of coherent structures in the recovery process, the three dimensional streamwise velocity and temperature iso-surfaces coloured by wallnormal distance are compared in Figs.15-17.
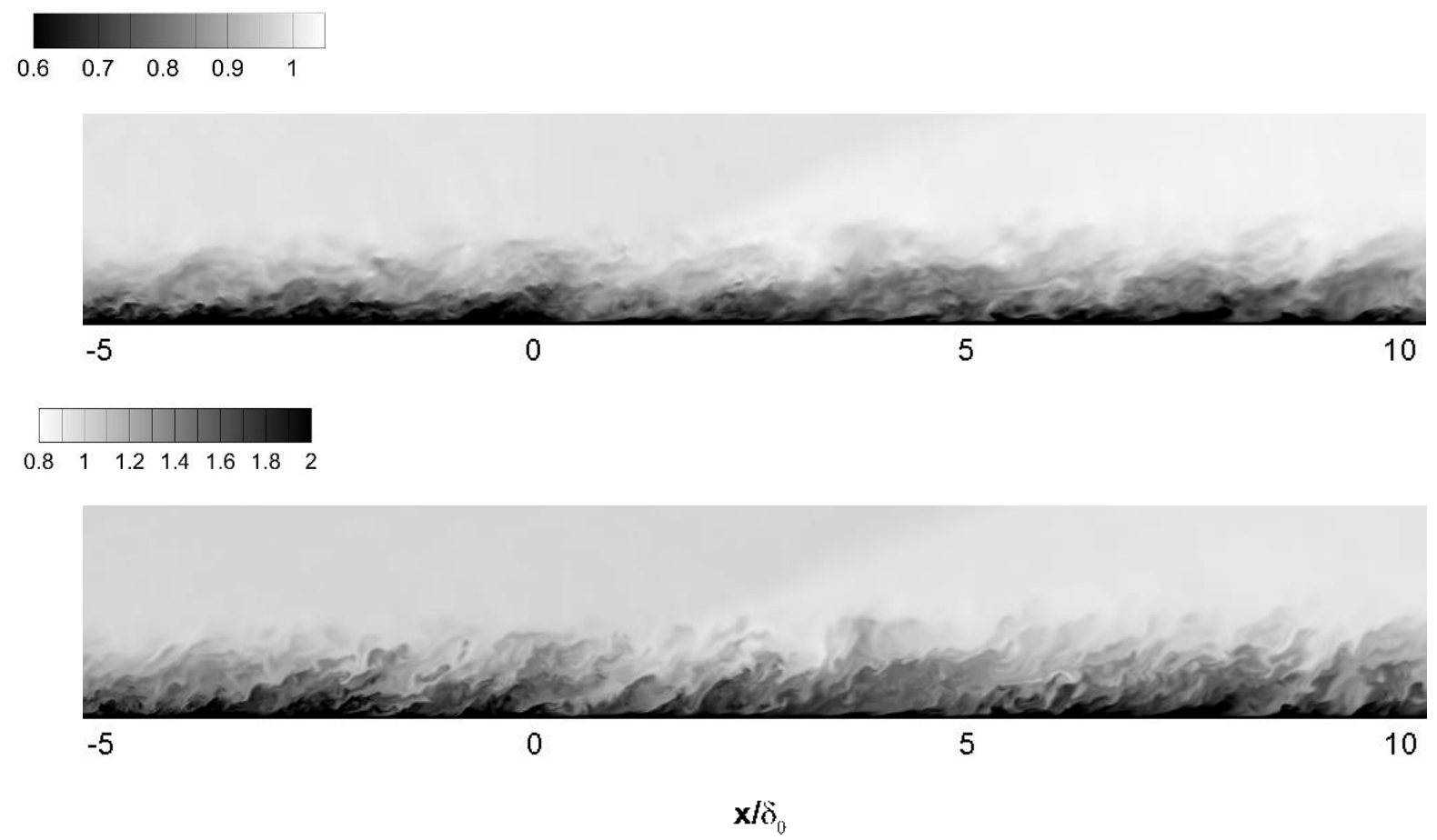

FIG.14 Turbulence coherent structures of $4^{\circ}$ expansion case visualized with the transformed velocity $u_{t}$ (top) $\left(u_{t}=u \cos \beta-v \sin \beta\right)$ and temperature (bottom).

For the flat plate case shown in Fig.15, we can clearly see the classic streamwise elongated vortices in the iso-surface of $u_{t}=0.3$, which keeps similar characteristics along the whole region. From the mean velocity profile shown in Fig.4, we can see that $u_{t}=0.3$ corresponds to an inner layer location. Smaller streaks disappear downstream of the corner. In the inner layer on the expansion ramp, the distance between two neighboring streaks increases. Turbulence mixing is reduced leading to the velocity deficiency in the inner layer illustrated in Fig.6.

The iso-surface of $u_{t}=0.55$ given in Fig.16b) shows the large scale structures in the outer boundary layer. The flat plate case shows that packets of large vortices develop and grow from the near-wall region to a higher wall-normal location. The interesting part of the structures is the packet that these iso-surfaces surround, which has lower velocity than that on the iso-surface. Those structures grow from the inner layer of the boundary layer and lift up to the outer layer. In the $4^{\circ}$ 
expansion case, as shown in Fig.16a), the flow suddenly accelerates across the expansion corner and the velocity profile becomes fuller in the outer layer, which smears all the small structures from upstream. From closer view of the contours of $u_{t}=0.55$, it is found that streaks containing lower velocity pockets from upstream are accelerated and the corrugated structures disappear in the high velocity region due to the expansion. The velocity fluctuation and the stress in this region is attenuated, indicating that the streaks from the inner part of the boundary layer disappear. Comparing the flat plate and the expansion case shows that the expansion modifies the velocity profile and weakens the lift-up process of the low energy streaks from the inner layer. Downstream of the expansion corner, at $x / \delta_{0}>5.0$, the coherent structures in the near wall region are regenerated during the recovery process and large-scale structures in the outer part of the boundary layer keep growing. Thus a strong exchange process exists between the inner layer and the outer layer. Combining Fig.15-16, we find that the streaks in the inner layer are not affected significantly by the expansion, while the vortex structures in the outer layer respond severely to the expansion. The near-wall quasi-streamwise vortices are both preserved, which indicates that the inner turbulence exists in a state of local equilibrium. Meanwhile in the outer layer of the boundary layer, the coherent structures on the ramp are affected by the upstream flow history, as well as the inner turbulence generation and its exchange with outer layer. The turbulence on the downstream ramp is especially affected by history effects of the slow turbulence decay downstream of the expansion corner. This determines formation of the two-layer structure in the boundary layer.

Temperature iso-surfaces of $T / T_{\infty}=2.1$ are shown in Fig. 17 . The evolution illustrated in Fig. 17 agrees well with the streamwise velocity field shown in Fig.15, which indicates that the temperature field is transported by the velocity field. The iso-surface is lifted and rolled into the outer layer, which reflects the vortices growing from inner layer and mixing with outer layer. 

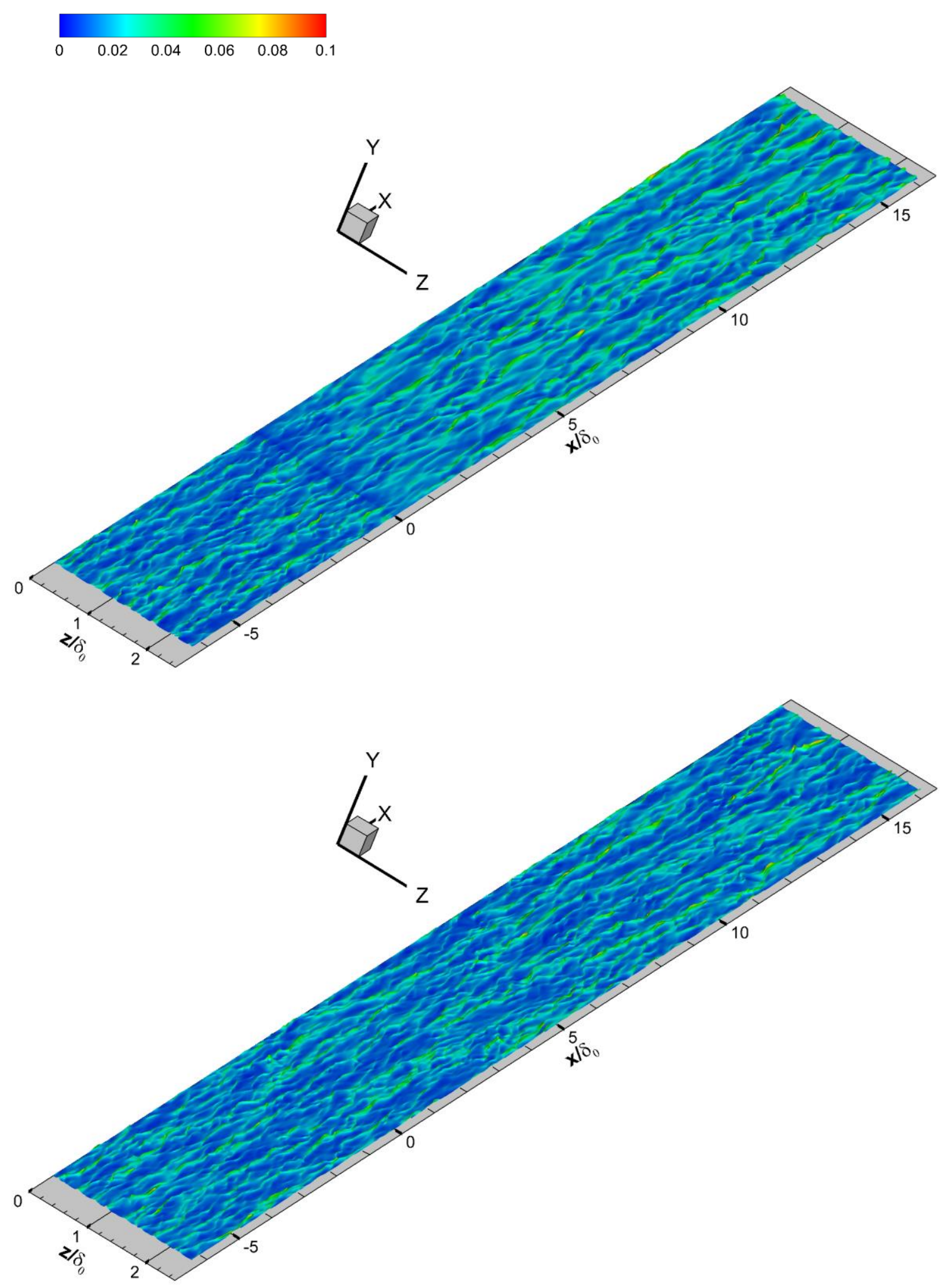

FIG.15 Turbulence coherent structures visualized with the iso-surface of $u_{t}=0.3$ and coloured by the wall-normal distance $y / \delta_{0}$ for the $4^{\circ}$ expansion case (top) and the flat plate (bottom). 

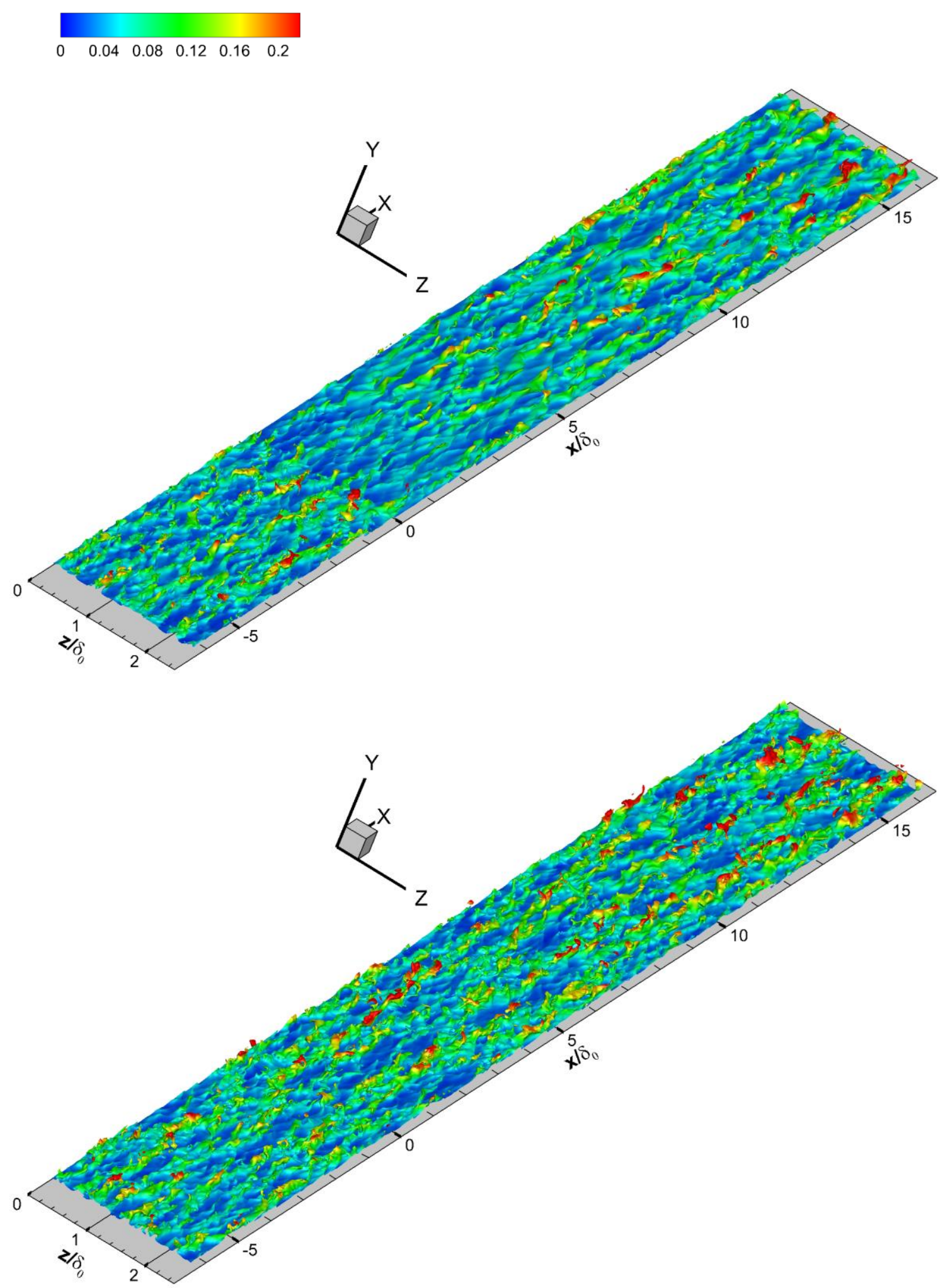

FIG.16 Turbulence coherent structures visualized with the iso-surface of $u_{t}=0.55$ and coloured by the wall-normal distance $y / \delta_{0}$ for the Expan 4 casae (top) and the flat plate (bottom). 


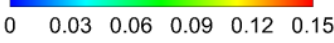
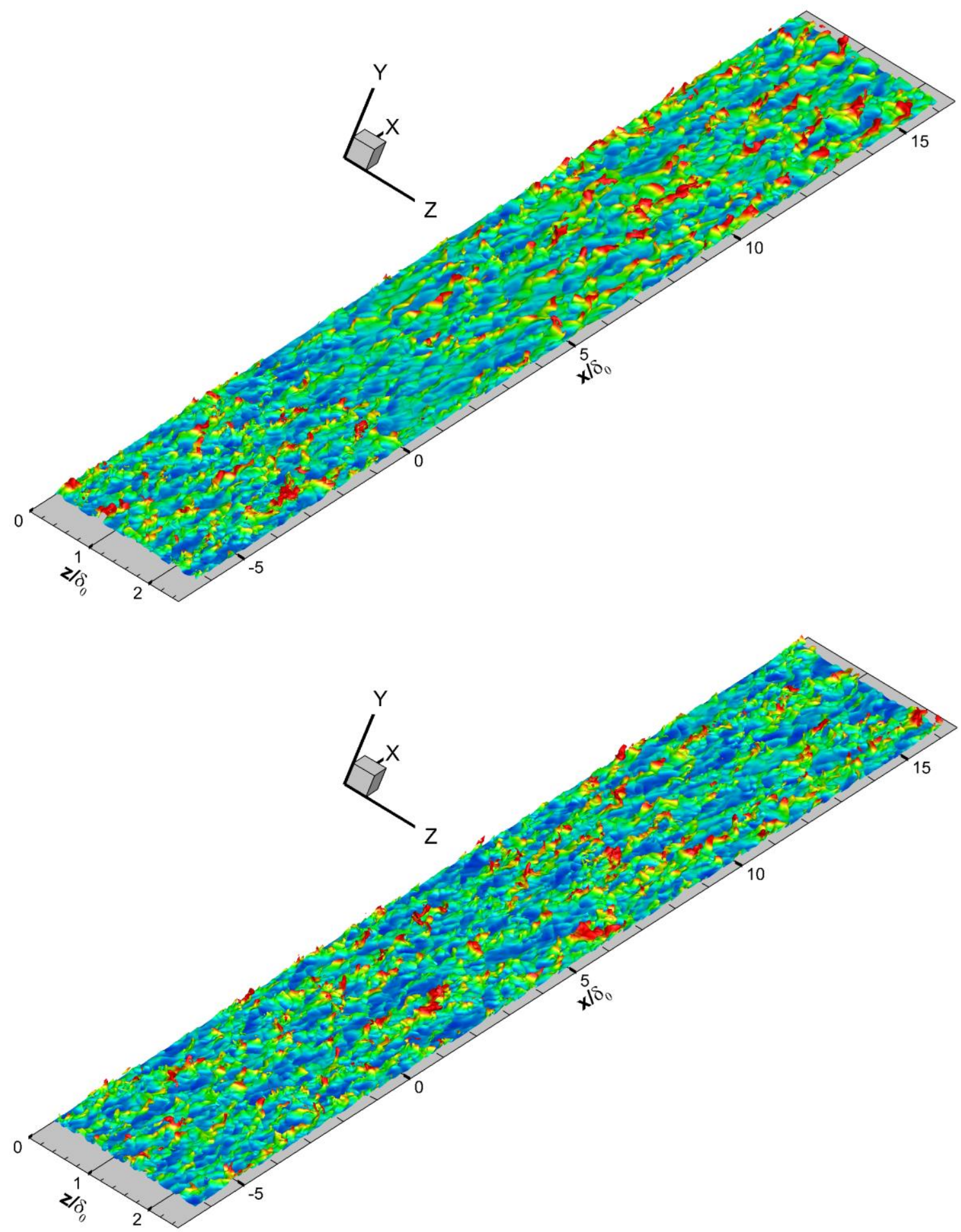

FIG.17 Turbulence coherent structures visualized with the iso-surface of non-dimensional temperature 2.1 and coloured by the wall-normal distance $y / \delta_{0}$ for the Expan 4 case (top) and the flat plate (bottom).

\section{Turbulence intensity and kinetic energy analysis}

\subsection{Turbulent intensity and kinetic energy distributions}


Fig.18 shows the evolution of the RMS velocity profiles along the streamwise direction for the flat plate and the $4^{\circ}$ expansion ramp. During the expansion process, all components of velocity fluctuations are suppressed. In the vicinity downstream of the corner (Fig. 18b), turbulence is suppressed significantly both in the inner layer and the outer layer. Turbulence recovers quickest in the inner layer. It matches the flat plate levels up to $y^{+}=40$ at $x / \delta_{0}=5.65$. Turbulence in the inner layer recovers fully to the same level as the flat plate flow at $x / \delta_{0}=15.35$. In the outer layer, turbulence is significantly suppressed along the expansion ramp, and the magnitudes of all velocity fluctuations are lower than the corresponding levels of the flat plate case throughout the domain. Comparing results of the $2^{\circ}$ expansion case at $x / \delta_{0}=15.35$ with the flat plate (Fig.19) shows that the supersonic flow has gone into a new turbulence equilibrium state, which is different from the flat plate. The reductions in turbulence level in the outer layer highlight a weakening of the large-scale structures across the expansion. An overall observation of Fig.18a)-d) is that the turbulence stresses in the expansion region present different modes of evolution in the inner layer and the outer layer regions, especially for $\left\langle u^{\prime} u^{\prime}\right\rangle$, which develops more quickly in the inner layer and the maximum of the peak is even higher than the flat plate case. For $\left\langle v^{\prime} v^{\prime}\right\rangle$ and $\left\langle w^{\prime} w^{\prime}\right\rangle$, the recovery is slower than $\left\langle u^{\prime} u^{\prime}\right\rangle$, which represents the turbulence energy redistributes in different directions and corresponds to the streak spacing change. Such a two-layer structure of turbulence is consistent with the experimental observation of Gillis and Johnston ${ }^{[2]}$ and the numerical simulation on the supersonic flow over a tandem expansion-compression corner by Fang et al. ${ }^{[22]}$.

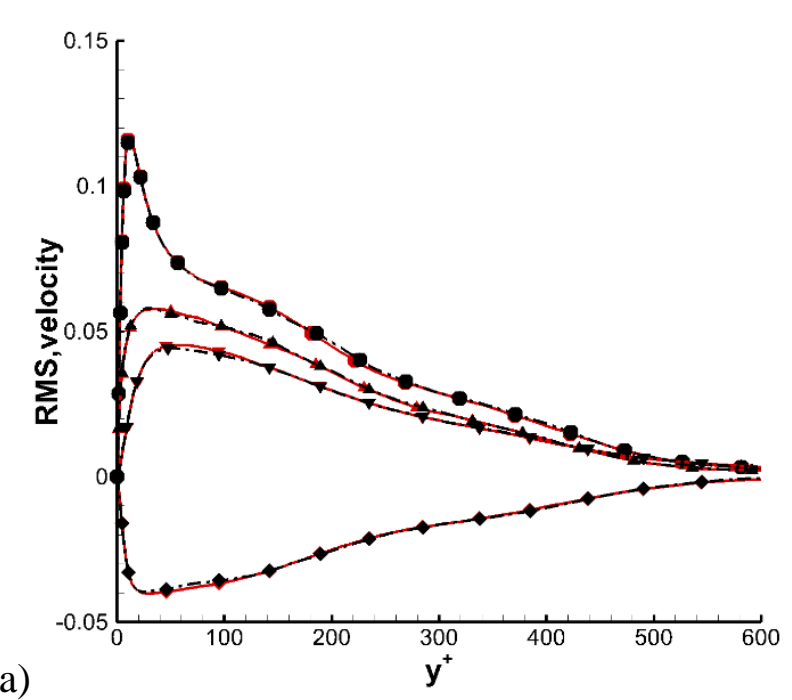

a)

b)

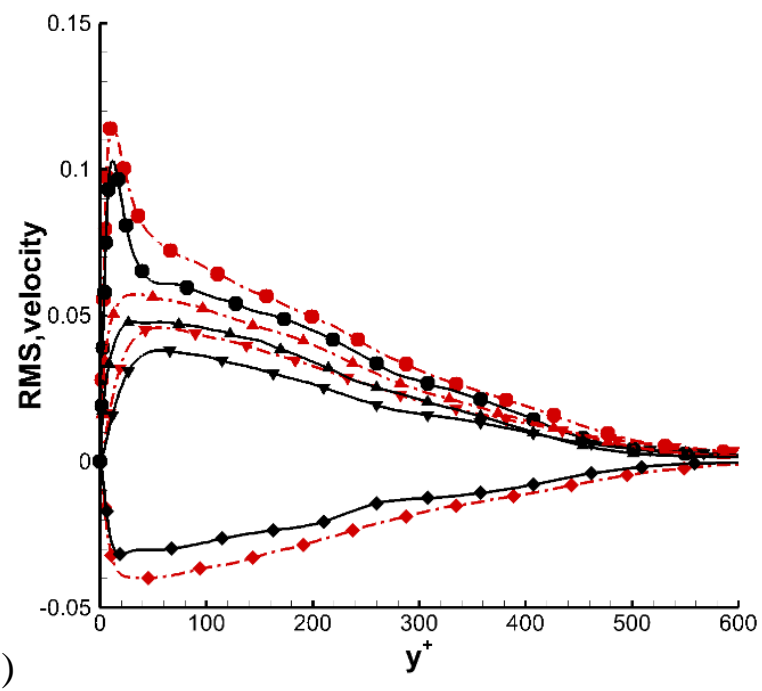



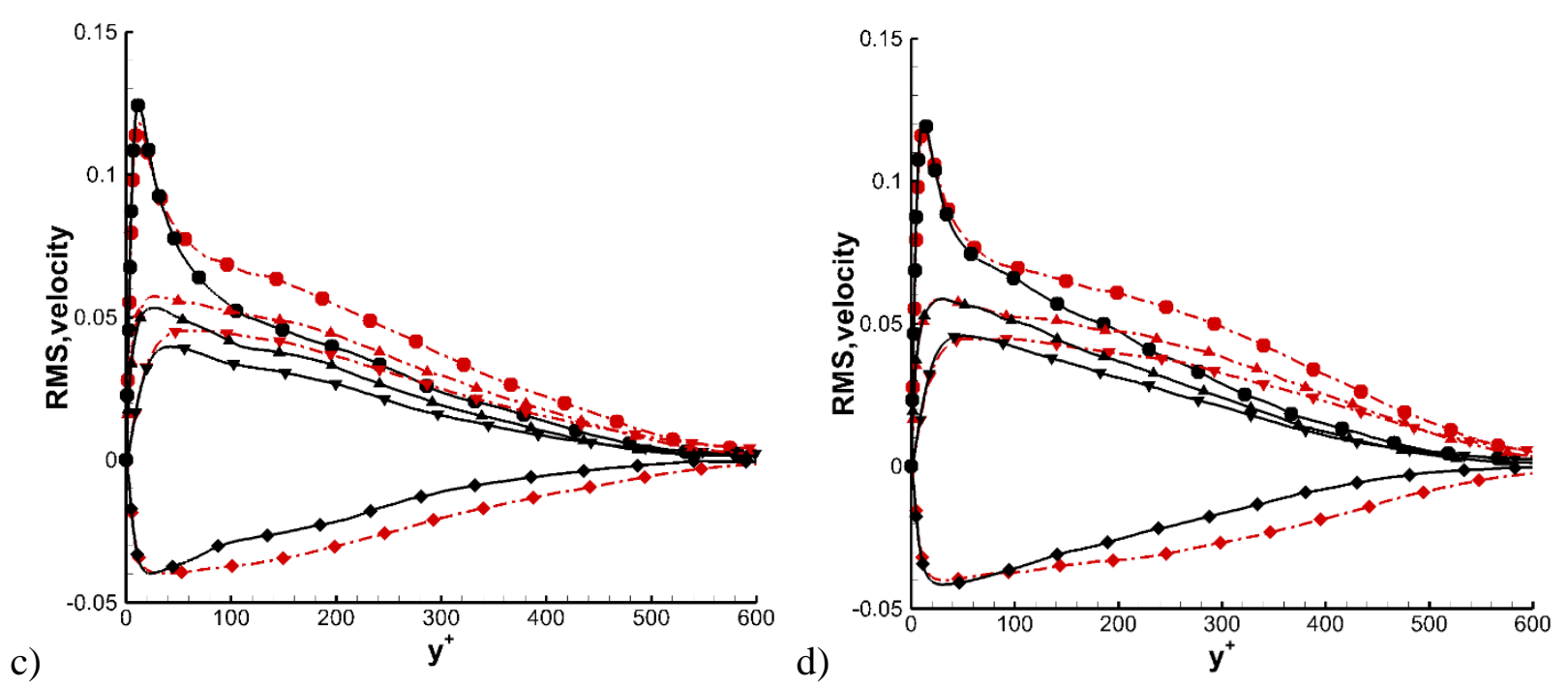

FIG.18 RMS velocity distributions at different streamwise locations for Expan4: solid (black) lines and the flat plate case: dashdotted (red) lines. RMS velocity of the streamwise $(\bullet)$, wall-normal $(\boldsymbol{\nabla})$, spanwise $(\boldsymbol{\Delta})$, of velocity fluctuations, together with Reynolds shear stress $(\diamond)$, a) $x / \delta_{0}=-2.42$, b) $x / \delta_{0}=0.81$, c) $x / \delta_{0}=5.65$, d) $x / \delta_{0}=15.35$.

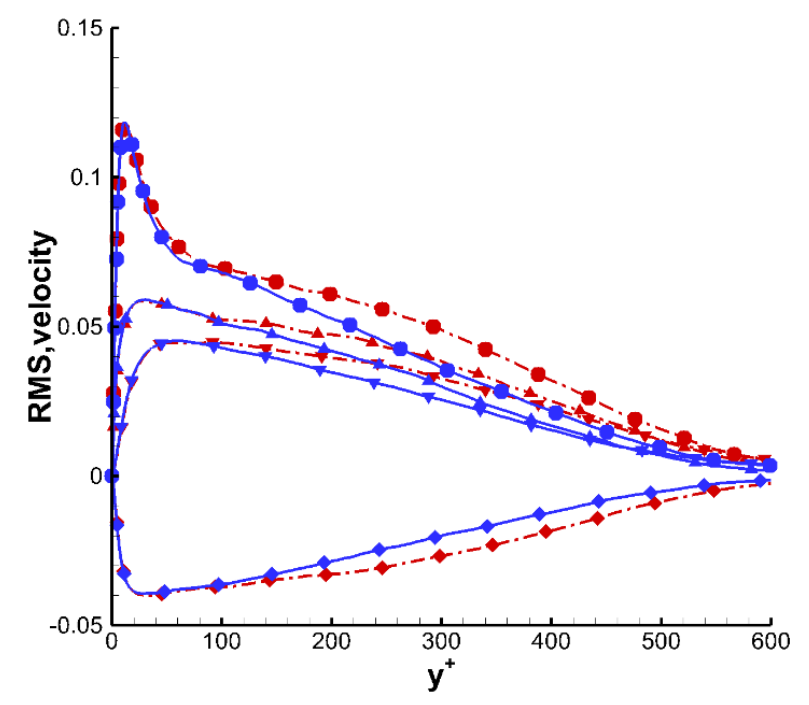

FIG.19 RMS velocity distributions at $x / \delta_{0}=15.35$, - solid line represents the flat plate, -. Dashdotted line represents Expan2. Symbols have the same meaning as in Fig.18.

TKE contours of flat plate and Expan 4 cases are plotted in Fig.20. It is found that TKE develops along the streamwise direction in flat plate case while TKE development is interrupted by the expansion in Expan 4 case, which exhibits a sudden reduction in the vicinity of the expansion corner and a recovery in the downstream region. Fig. 21 gives the TKE plots along the streamwise direction at different wall-normal locations which correspond to streaks shown in Fig.11. It is found that TKE in the inner layer $\left(y / \delta_{0}=0.0266\right)$ demonstrates a quick recovery than the outer layer and TKE recovery gets slower when the wall-normal location is larger. Certainly this is a coarse estimation 
since the TKE profile is related to $y^{+}$instead of $y / \delta_{0}$ and $y^{+}$changes along expansion ramp when same $y / \delta_{0}$ is set with flat plate case.

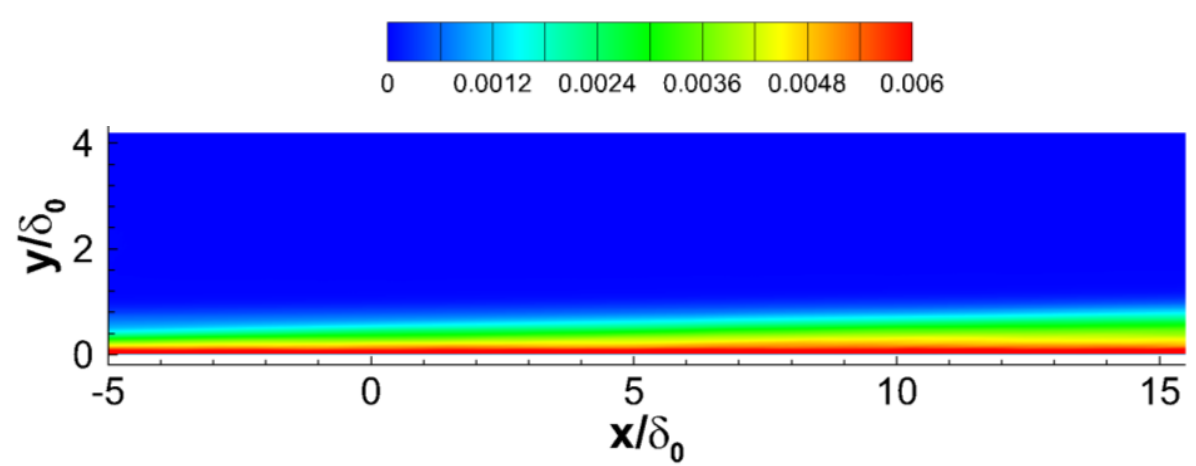

a) Flat plate

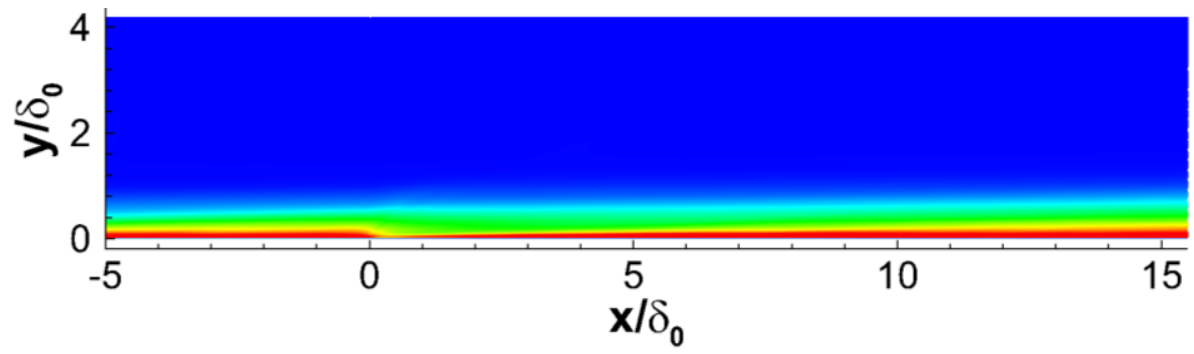

b) Expan 4

FIG.20 TKE contour comparison between the flat plate and the Expan4 case.

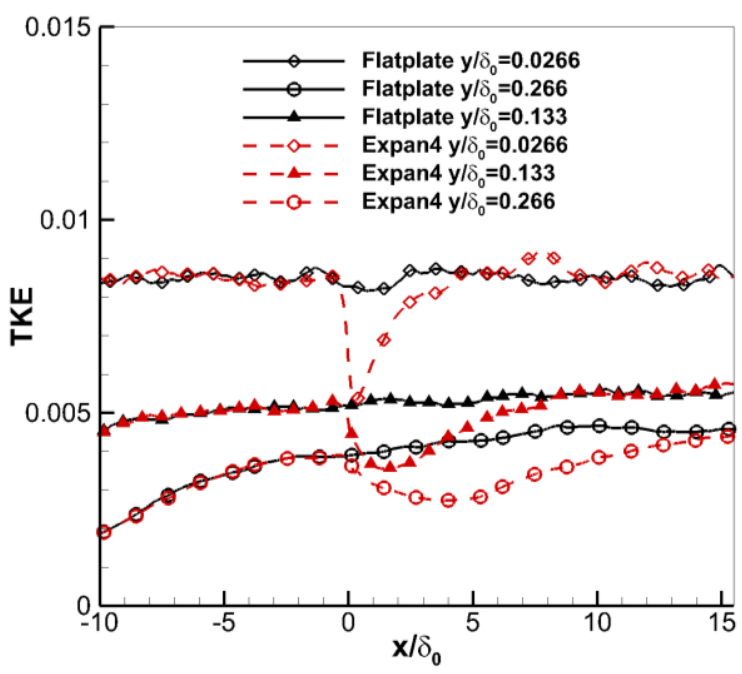

FIG.21 TKE vs $\mathrm{x} / \delta_{0}$ at different $y / \delta_{0}$ locations of the flat plate and the Expan 4 case. 


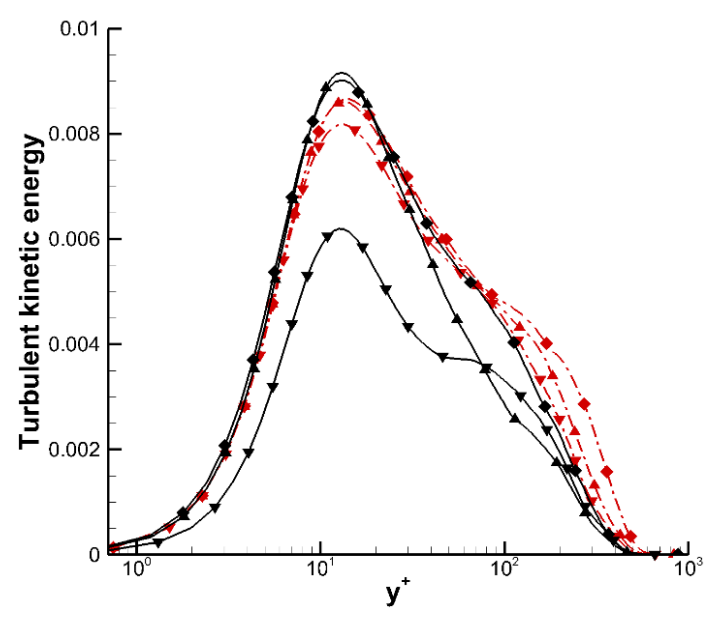

FIG.22 TKE profiles of the flat plate (dashdotted line) and Expan4 (solid line) at different streamwise locations, including $x / \delta_{0}=0.81($ symbol $\boldsymbol{\nabla}), x / \delta_{0}=5.65$ ( symbol $\left.\boldsymbol{\Delta}\right)$,

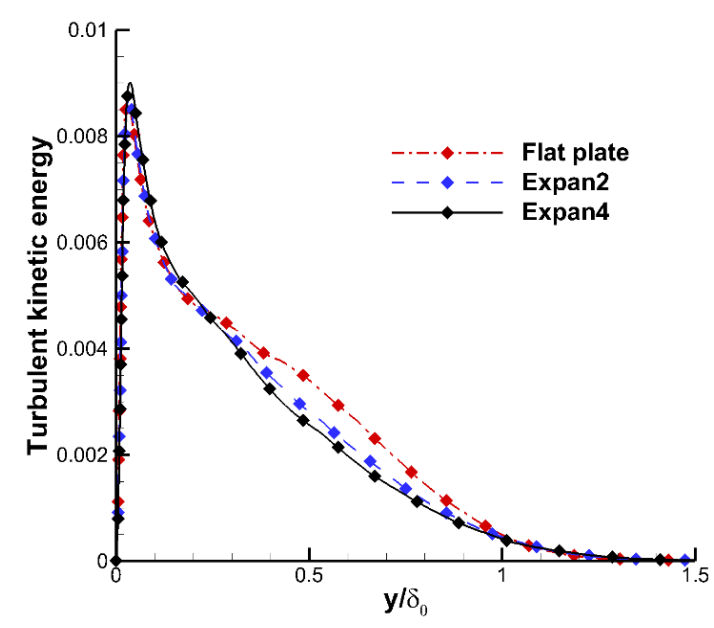

FIG.23 TKE profile comparison of the flat plate (dashdotted line), Expan2 (dashed line) and Expan4 (solid line) at $x / \delta_{0}=15.35$. $x / \delta_{0}=15.35$ (symbol

TKE profiles at different streamwise locations of all cases vs $y^{+}$are shown in Fig.22. As would be expected, a sharp reduction in TKE occurs immediately downstream of the corner. In addition, the TKE profile gradually recovers to the equilibrium profile downstream of the corner. Fig.22 clearly demonstrates a two-layer structure. Turbulence across the corner is suppressed significantly in both the inner and outer layers. Downstream of the corner, the TKE in the inner layer recovers quickly and even exceeds the flat plate level on the same streamwise location. In contrast, the outer boundary layer suffers an apparent turbulence suppression. TKE profile comparison of the flat plate, $2^{\circ}$ and $4^{\circ}$ expansion cases at $x / \delta_{0}=15.35$ are shown in Fig. 23. It is seen that the turbulence intensity reduces in the Expan2 case. The dividing line is located at $y^{+} \in[50,100]$ or $y / \delta_{0} \in[0.11,0.22]$. Recalling the Reynolds shear stresses shown in Fig.18, the reductions in turbulence level in the outer layer highlight a weakening of the large-scale structures across the expansion. We deduce that due to weakened turbulence in the outer layer, the turbulence exchange of inner layer with mainstream is greatly suppressed, which results in a turbulent energy accumulation in the inner layer since turbulence in the inner layer exists in a local equilibrium and its production is still ongoing. The turbulence energy in the inner layer cannot transfer through the outer layer since the suppressed exchange in the outer layer blocks the transfer. In summary, the two-layer structure is due to the decay of the turbulence shear stress in the log-law region of the boundary layer 
downstream of expansion corner, which reduces the exchange between the turbulent structures in the inner layer and the mainstream.

\subsection{Turbulent Mach number}

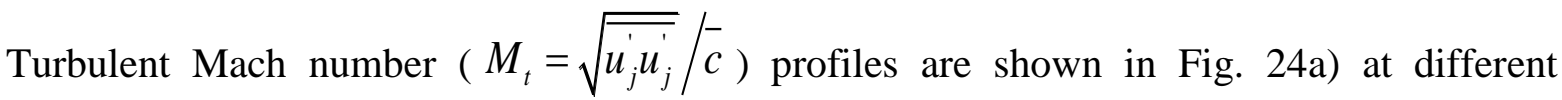
streamwise locations. In addition to the turbulent Mach number, we also examine the fluctuating Mach number $M^{\prime}\left(=\left(\overline{M^{2}}-\bar{M}^{2}\right)^{1 / 2}\right.$, Fig. 24b), since it presents different information in compressible flows. The turbulent Mach number is no more than 0.3 and its maximum peak is located near the wall. For the flat plate case, the fluctuating Mach number $M^{\prime}$ starts to build a second local peak in the outer part of the boundary layer. This peak moves away from the wall further downstream, as can be seen in Fig. 24b). In the region just downstream of the corner, both the turbulent Mach number and the fluctuating Mach number show a sudden decrease. In the inner layer, both the turbulent Mach number and the fluctuating Mach number increase rapidly downstream after the sudden drop at the corner. The first peak overshoots the flat plate case. In the outer layer, turbulent Mach number and $M^{\prime}$ decrease compared to the flat plate case. For $M^{\prime}$, the second peak is greatly weakened due to the suppression of turbulence.

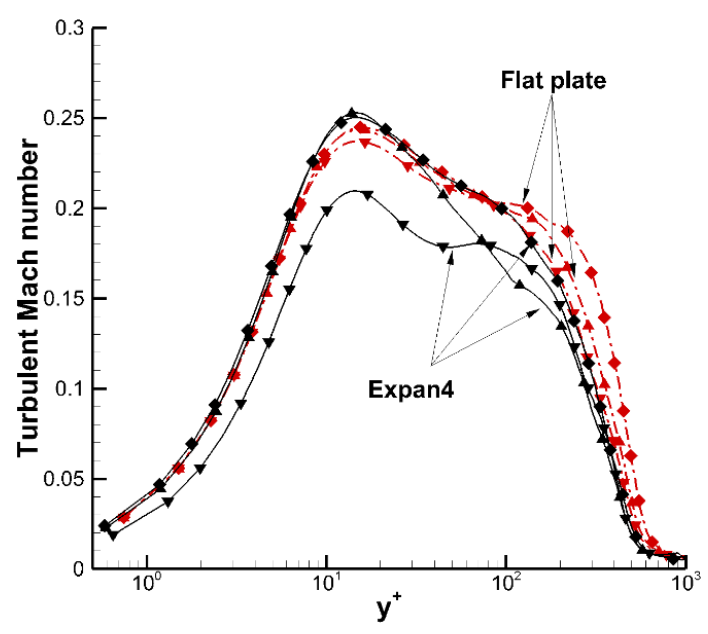

a) Turbulent Mach number

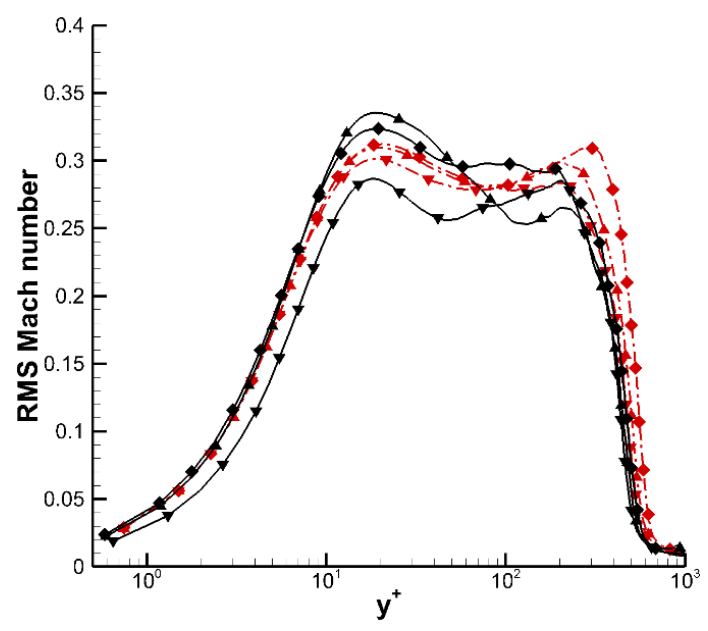

b) Fluctuating Mach number

FIG.24 Profiles of the turbulent Mach number $M_{t}$ and the fluctuating Mach number $M^{\prime}$ of the flat plate (dashdotted line) and Expan4 (solid line) at different streamwise locations, including $x / \delta_{0}=0.81$ (symbol $\left.\boldsymbol{\nabla}\right), x / \delta_{0}=5.65$ (symbol $\boldsymbol{\Delta}$ ), $x / \delta_{0}=15.35$ (symbol 


\subsection{Turbulent kinetic energy budgets}

To further assess the boundary layer on the expansion ramp, the budget of the TKE was analyzed and compared to the flat plate results, as shown in Fig.25. The TKE is defined as $\tilde{k}=\overline{\rho u_{i}^{\prime \prime} u_{i}^{\prime \prime}} / 2 \bar{\rho}$, where the superscript refers to fluctuations from the Favre averages, commonly used to simplify the resulting energy equation given by

$$
\begin{aligned}
& \frac{\partial}{\partial t}(\bar{\rho} \bar{k})+\tilde{u}_{j} \frac{\partial}{\partial x_{j}}(\bar{\rho} \bar{k})=P+T+I I+D-\phi+V_{c} \\
& P=-\overline{\rho u_{i}^{\prime \prime} u_{j}^{\prime \prime}} \frac{\partial \tilde{u}_{i}}{\partial x_{j}} \\
& T=-\frac{1}{2} \frac{\partial}{\partial x_{j}} \overline{\rho u_{i}^{\prime \prime} u_{i}^{\prime \prime} u_{j}^{\prime \prime}} \\
& I I=I I_{t}+I I_{d}=-\frac{\partial}{\partial x_{j}} \overline{p^{\prime} u_{i}^{\prime \prime}} \delta_{i j}+\overline{p^{\prime} \frac{\partial u_{i}^{\prime \prime}}{\partial x_{i}}} \\
& D=\frac{\partial}{\partial x_{j}} \overline{\frac{u_{l}^{\prime \prime}}{R e} \tau_{l j}^{\prime}} \\
& \phi=\overline{\frac{1}{\operatorname{Re}} \frac{\partial u_{i}^{\prime \prime}}{\partial x_{j}} \tau_{i j}^{\prime}} \\
& V_{c}=-\overline{u_{j}^{\prime \prime}} \frac{\partial \bar{p}}{\partial x_{j}}+\frac{\overline{u_{i}^{\prime \prime}}}{\operatorname{Re}} \frac{\partial \overline{\tau_{i l}}}{\partial x_{l}}-\bar{\rho} \bar{k} \frac{\partial \tilde{u}_{j}}{\partial x_{j}} \\
& C=-\tilde{u}_{j} \frac{\partial}{\partial x_{j}}(\bar{\rho} \bar{k})
\end{aligned}
$$

Here $P$ is the production term, showing the rate of generation of TKE by velocity gradients, $T$ is the turbulent transport, $I I$ are the pressure terms, including the pressure diffusion $\left(I I_{t}\right)$ and the pressure dilatation $\left(I I_{d}\right), D$ is the viscous diffusion, $-\phi$ is the pseudo-viscous dissipation and $C$ is the convective term. $V_{c}$ includes the terms that arise when the density is not constant. The explicit forms of the different terms are given by Guarini et al. ${ }^{[15]}$.

Fig.25 compares the distributions of these terms, after normalization by the wall quantity $\rho_{w} u_{\tau}^{4} / \nu_{w}$, at all streamwise locations. $V_{c}$ is small (its maximum is 28 times smaller than the production term in the present calculations on the $4^{\circ}$ expansion ramp) therefore has not been 
included on the plot for clarity. Its maximum is a factor of 28 smaller than that of the production term in the present calculations on the $4^{\circ}$ expansion ramp. At $x / \delta_{0}=-2.42$ upstream of the corner, the TKE budget shows a typical behavior of a boundary layer at zero-pressure gradient, in which production is balanced by dissipation over most of the boundary layer. Viscous diffusion is only important in the near wall region. In the viscous sub-layer, production becomes negligible and diffusion is balanced by dissipation. Near the expansion corner at $x / \delta_{0}=0.81$, all terms are greatly changed, especially the production and dissipation terms, which are greatly reduced in the vicinity of expansion corner. In the inner region all terms follow the general shape of the flat plate case but with smaller amplitude. However, the relationship of all terms becomes much more complicated in the outer part. TKE production significantly decreases in the outer layer $\left(y^{+} \in[60,500]\right)$ and even changes sign in the region of $y^{+} \in[220,330]$, which drives the large reduction of the turbulence in the outer layer. The dissipation is almost unchanged and can be neglected. The convection term $C$ at the boundary layer edge becomes positive and the magnitude increases greatly in the outer boundary layer at $x / \delta_{0}=0.81$. This is because the local wall normal velocity increases almost $5 \sim 7$ times more than the flat plate case due to the expansion effects. The transport term is suppressed immediately downstream of the expansion and becomes negative near the boundary layer edge, which implies that weakened turbulence in the outer layer of the boundary layer promotes less turbulent transport. Recalling the analysis in Section 4.3, we conclude that these attenuated vortices decrease the production and the transfer of the TKE. TKE pressure diffusion $\left(I I_{t}\right)$ and dilatation $\left(I I_{d}\right)$ term $I I$ becomes more significant due to the sudden favourable pressure gradient caused by the rapid expansion. Here we consider the contribution of the pressure terms, $I I_{t}$ and $I I_{d}$. Fig.26 shows the ccomparison of pressure terms at $\mathrm{x} / \delta_{0}=0.81$ between the $4^{\circ}$ expansion and flat plate cases. The pressure diffusion term is larger than the pressure dilatation term, which indicates that the pressure diffusion effect plays a dominant role instead of the compressibility effect.

At $x / \delta_{0}=5.65$, all TKE budget terms have almost recovered to the undisturbed flat plate boundary layer in the inner part, while the terms in the outer layer still deviate from the flat plate case, which confirms that turbulence in the inner part recovers quicker than in the outer part. At $x / \delta_{0}=15.35$ the TKE budgets show that turbulence in the outer part recovers slowly towards an equilibrium state, with the wall region $y^{+}<150$ having recovered. 
At $x / \delta_{0}=5.65$ the production is almost the same as the flat plate case in the inner layer, while the term in the outer layer is still far lower than the flat plate case and the negative production vanishes. At $x / \delta_{0}=15.35$ the production term in the outer layer increases in intensity, which reflects the regeneration of the wall-turbulence and the recovery of the boundary layer toward equilibrium. However, the production term of expansion is still lower than that on flat plate due to the upstream history effects.

The convective term and transport term at $x / \delta_{0}=5.65$ and $x / \delta_{0}=15.35$ are both smaller than in the undisturbed flat plate boundary layer, which indicates that these terms in the outer layer inhibit spreading of the increasing TKE in the inner part to spread out. Hence, the long recovery in the outer part of the boundary layer can be explained as a combined effect of decay of turbulence in the outer layer and the transport restraint of the outer layer. The exchange between the turbulent structures in the inner layer and the mainstream is blocked by the combined effect and finally leads to a two layer structure, which is illustrated by a quick recovery in the inner layer and a slow recovery in the outer layer.
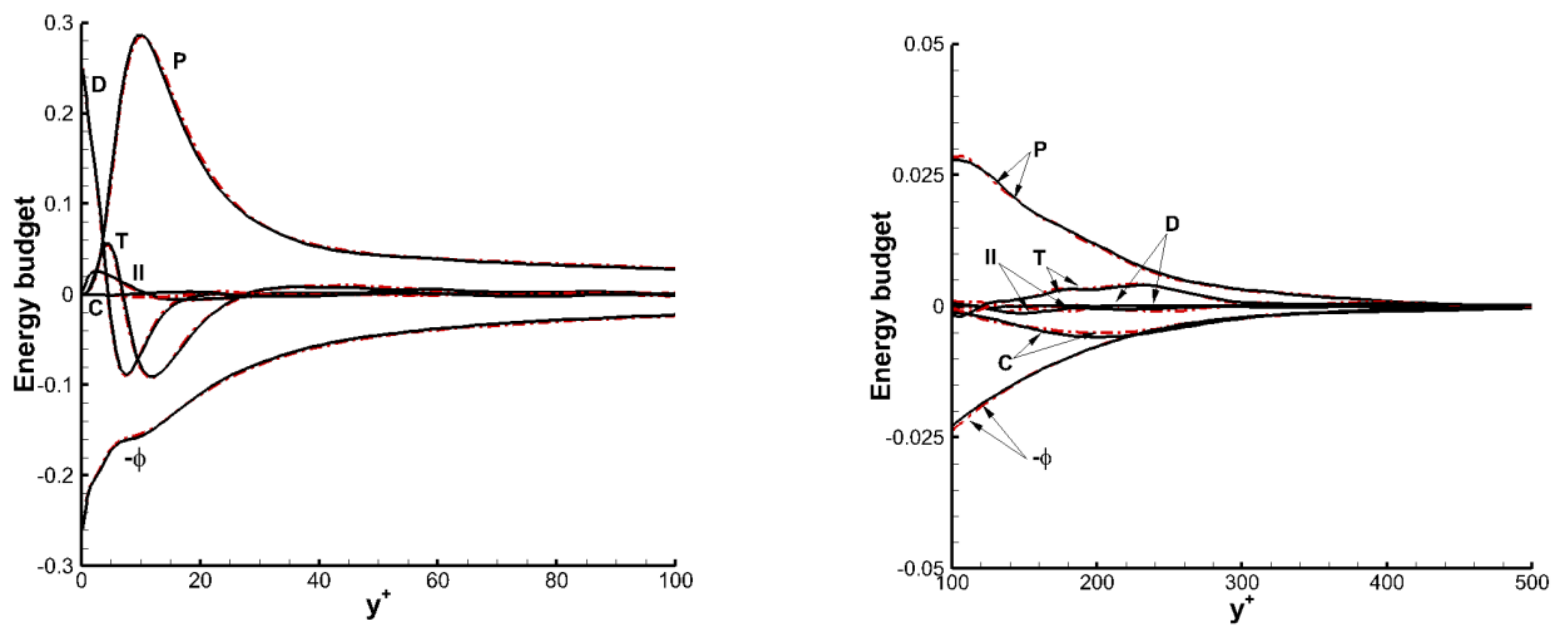

a) $x / \delta_{0}=-2.42$ 

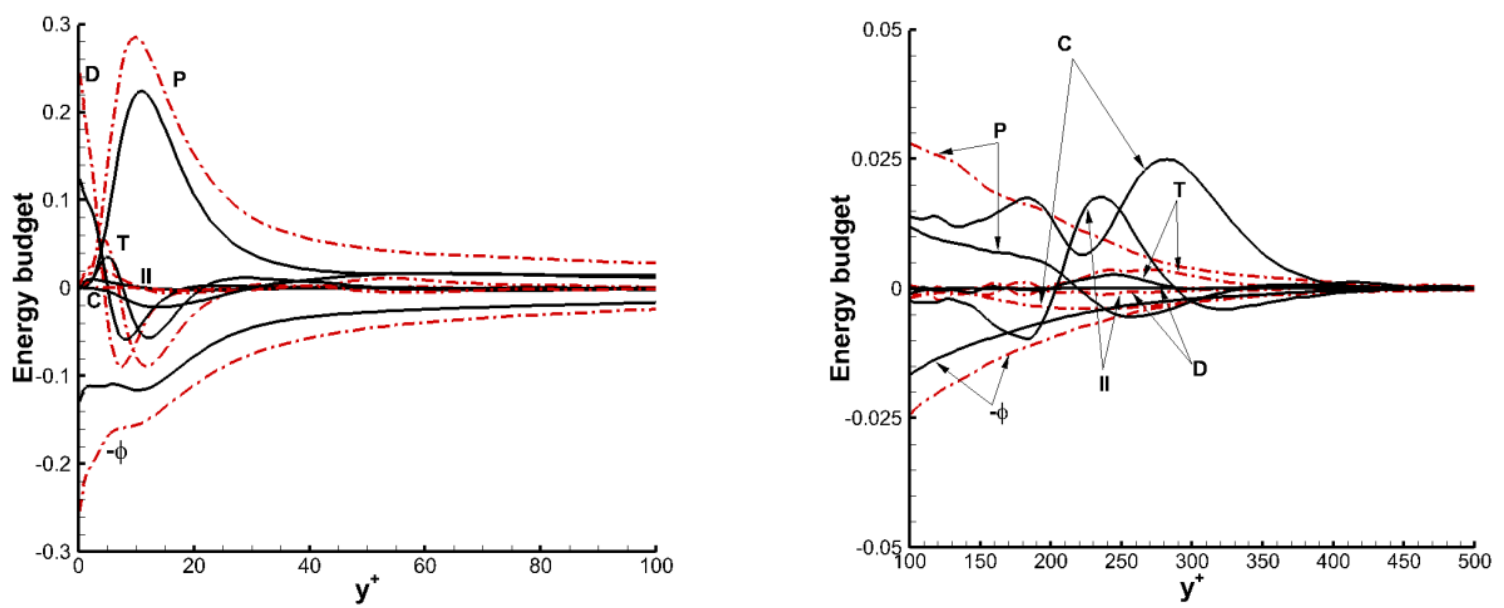

b) $x / \delta_{0}=0.81$
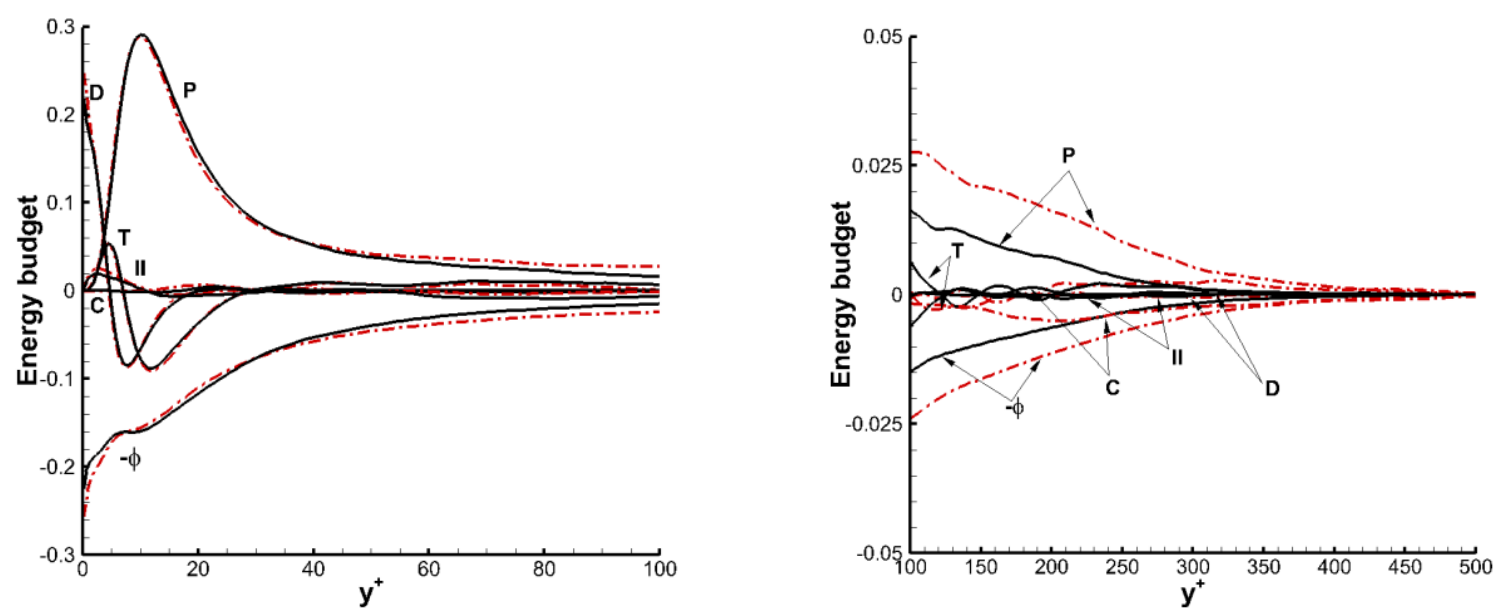

c) $x / \delta_{0}=5.65$
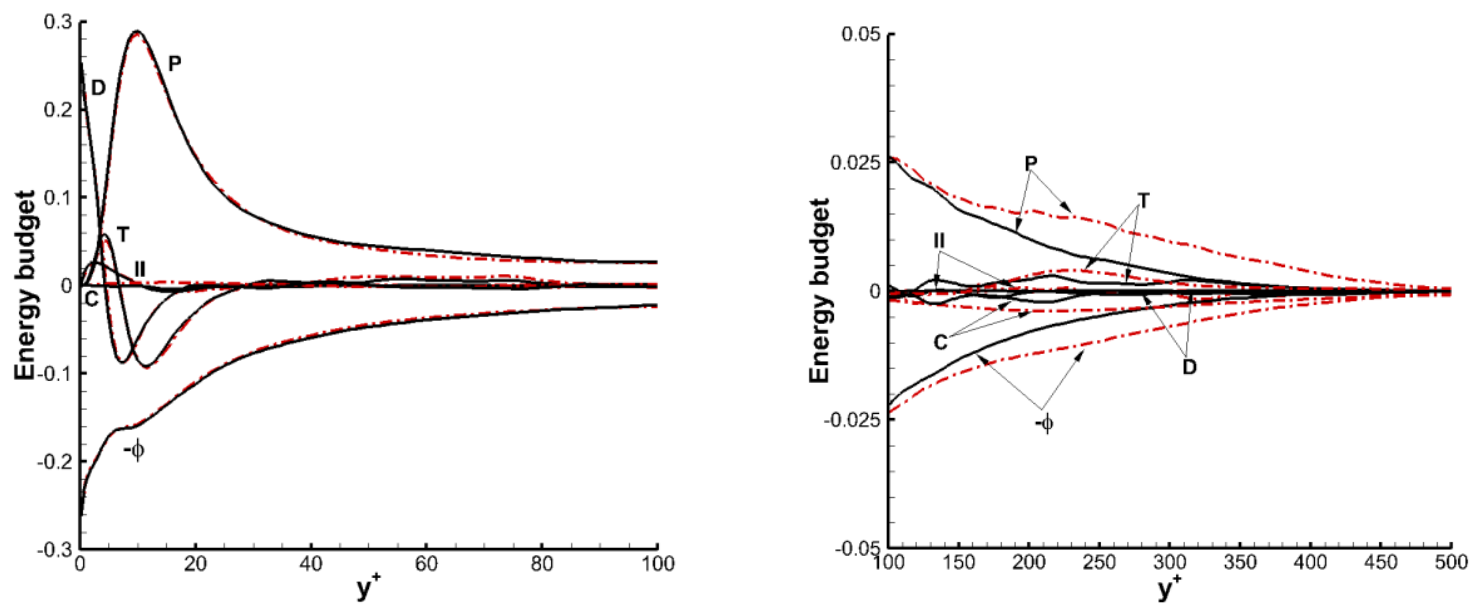

d) $x / \delta_{0}=15.35$

FIG.25 TKE budget profiles of the flat plate and Expan4 at different streamwise locations, a) $x / \delta_{0}=-2.42$, b) $x / \delta_{0}=0.81$,

c) $x / \delta_{0}=5.65$, d) $x / \delta_{0}=15.35$, - solid line represents $4^{\circ}$ expansion case, -. Dashdotted line represents the flat plate. 

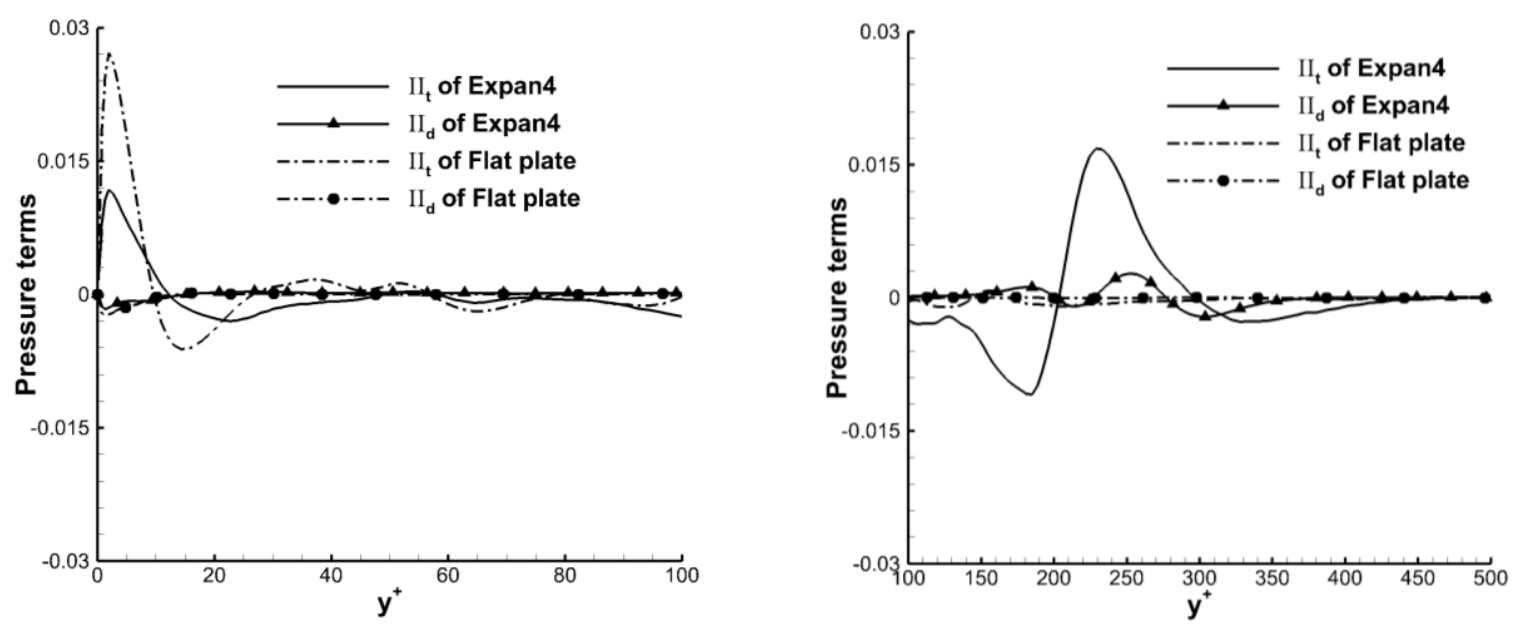

FIG.26 Comparison of pressure terms of flat plate case and $4^{\circ}$ expansion case at $x / \delta_{0}=0.81$.

\section{Conclusions}

Direct numerical simulations have been carried out to investigate an $M=2.7$ supersonic TBL over expansion corners with deflection angles of $0^{\circ}$ (flat plate), $2^{\circ}, 4^{\circ}$. Turbulence properties in the recovery flowfield are analyzed in detail. The skin friction, pressure, velocity profile and boundary layer thickness growth demonstrate non-equilibrium and equilibrium states downstream of the corner. The streamwise velocity reduces in the near wall region, even though the main velocity increases compared to flat plate case. A shape factor is introduced to evaluate the velocity profile fullness of the boundary layer. A higher shape factor value occurs in the expansion cases, which implies that the supersonic flow over expansion ramp has a decreased resistance to an adverse pressure gradient.

Turbulence structures on the expansion ramp are clearly visible, including $\lambda_{2}$ vortices, streaks, and iso-surfaces of streamwise velocity and temperature. Turbulence is suppressed to a large extent downstream of the corner and this status remains in the recovery region even though turbulence is regenerated in the near wall region. The two-point correlation function in the homogenous spanwise direction demonstrates a larger spanwise streak spacing for expansion flows compared to the flat plate. TKE profiles and budgets along the streamwise direction exhibit a characteristic two-layer structure in the expansion cases. Compared to the outer layer, turbulence in the inner layer recovers to equilibrium more quickly and exists in an approximate balance of the local production and dissipation. In the outer layer, turbulence is consistently suppressed along the ramp and recovers to an equilibrium state far downstream. The two-layer structure is found to be 
due to history effects of the turbulence decay in the outer part of the boundary layer downstream of the expansion corner, with limited the momentum and energy exchange between the inner layer and the mainstream and finally leads to a quick recovery in the inner layer and a slow recovery in the outer layer.

\section{Acknowledgement}

Supercomputer time provided by the UK Engineering and Physical Sciences Research Council under the project 'Thermal and Reactive Flow Simulation on High-End Computers' (Grant No. $\mathrm{EP} / \mathrm{J} 016381 / 1)$ is gratefully acknowledged.

This work was also supported by grants from the National Natural Science Foundation of China (11472305, 91541101 and 11522222), and an outstanding youth fund of National University of Defense Technology.

\section{Reference}

[1] Knight D D, Yan H, Panaras A G, Zheltovodov A A. Advances in CFD prediction of shockwave turbulent boundarylayer interactions. Prog. Aerosp. Sci. 39, 121-184 (2003)

[2] Gillis J, Johnston J. Turbulent boundary-layer flow and structure on a convex wall and its redevelopment on a flat wall. J. Fluid Mech. 135, 123-153 (1983)

[3] Goldfeld M A, Nestoulia R V, A. N. Shiplyuk. Re-laminarization of a turbulent boundary layer with a Mach number $\mathrm{M}=4$. J. Appl. Mech. Tech. Phys. 43 (1), 76-82 (2002)

[4] Dussauge J, Gaviglio J. The rapid expansion of a supersonic turbulent flow: role of bulkdilatation. J. Fluid. Mech. 174, 81-112 (1987)

[5] So R, Mellor G. Experiment on convex curvature effects in turbulent boundary layers J. Fluid. Mech. 60, 43$62(1973)$

[6] Tichenor N, Humble R, Bowersox R. Response of a hypersonic turbulent boundary layer to favourable pressure gradients. J. Fluid Mech. 722, 187-213 (2013)

[7] Smith D, Smits A. The rapid expansion of a turbulent boundary layer in a supersonic flow. Theor. Comput. Fluid Dyn. 2 (319-328), (1991)

[8] Arnette S, Samimy M, Elliott G. Structure of supersonic turbulent boundary layer after expansion regions. AIAA J. 33 (3), 430-437 (1995)

[9] Arnette S, Samimy M, Elliott G. The effects of expansion on the turbulence structure of a compressible boundary layer. J. Fluid. Mech. 367, 67-105 (1998)

[10] Dawson J, Samimy M, Arnette S. Effects of expansions on supersonic boundary layer: Surface pressure measurements. AIAA J. 32 (11), 2169-2177 (1994)

[11] Chung K. Interaction region of turbulent expansion corner flow. AIAA J. 36 (6), 1115-1116 (1998)

[12] Zheltovodov A, Schulein E, Horstman C. Development of separation in the region where a shockinteracts with a turbulent boundary layer perturbed by rarefaction waves. J. Appl. Mech. Tech. Phys. 34 (3), 346-354 (1993) 
[13] Gao T Y, Liang J H, Sun M B, Zhong Z. Dynamic combustion characteristics in a rectangular supersonic combustor with single-side expansion. Proc. IMechE. Part G: J. Aerospace Engineering doi: 10.1177/0954410016662062, (2016)

[14] Nguyen T T Q, Behr M, Reinartz B U. Numerical Investigation of Compressible Turbulent Boundary Layer Over Expansion Corner. AIAA paper 2009-7371, 2009

[15] Guarini S E, Moser R D, Shariff K, Wray A. Direct numerical simulation of a supersonic boundary layer at Mach 2.5. J. Fluid Mech. 414, 1-33 (2000)

[16] Pirozzoli S, Grasso F, Gatski T B. Direct numerical simulation and analysis of a spatially evolving supersonic turbulent boundary layer at Ma2.25. Phys. Fluids 16 (3), 530-545 (2004)

[17] Martin M. Direct numerical simulation of hypersonic turbulent boundary layers. Part 1. Initialization and comparison with experiments. J. Fluid Mech. 570 (347-364), (2007)

[18] Lagha M, Kim J, Eldredge J D, Zhong X. A numerical study of compressible turbulent boundary layers. Phys. Fluids 23 (015106), (2011)

[19] Touber E. Unsteadiness in shock wave boundary layer interactions. PhD thesis, University of Southampton, 2010

[20] Sandham N D, Schülein E, Wagner A, Willems S, Steelant J. Transitional shock-wave/boundary-layer interactions in hypersonic flow. J. Fluid Mech. 752, 349-382 (2014)

[21] Wu M, M. P. Martin. Direct Numerical Simulation of Supersonic Turbulent Boundary Layer over a Compression Ramp. AIAA J. 45 (4), 879-889 (2007)

[22] Fang J, Yao Y F, Zheltovodov A A, Li Z R, Lu L P. Direct numerical simulation of supersonic turbulent flows around a tandem expansion-compression corner. Phys. Fluids 27, 125104 (2015)

[23] Touber E, Sandham N D. Large-eddy simulation of low-frequency unsteadiness in a turbulent shock-induced separation bubble. Theor. Comput. Fluid Dyn. 23, 79-107 (2009)

[24] Xie Z T, Castro I P. Efficient generation of inflow conditions for large-eddy simulation of street-scale flows. Flow Turbul. Combust. 81, 449-470 (2008)

[25] Wang B, Sandham N D, Hu Z, W Liu. Numerical study of oblique shock-wave/boundary-layer interaction considering sidewall effect. J. Fluid Mech. 767, 526-561 (2015)

[26] Schlatter P, Orlu R. Assessment of direct numerical simulation data of turbulent boundary layers. J. Fluid. Mech. 659, 116-126 (2010)

[27] Sun M B, Zhang S P, Zhao Y H, Zhao Y X, Liang J H. Experimental investigation on transverse jet penetration into a supersonic turbulent crossflow. Sci. China Tech. Sci. 56 (8), 1989-1998 (2013)

[28] Bradshaw P. Effects of streamline curvature on turbulent flow. AGARD AG-169, 1973

[29] Morkovin M V. Effects of compressibility on turbulent flows, in In Mecanique de la Turbulence (ed. A. Favre) CNRS, pp. 367-380, 1962

[30] Duan L, Beekman I, Martin M P. Direct numerical simulation of hypersonic turbulent boundary layers. Part 3. Effect of Mach number. J. Fluid Mech. 672, 245-267 (2011)

[31] White F M. Viscous Fluid Flow. McGraw Hill, 3rd edition, 2006

[32] Young A D. Boundary Layers - AIAA Education Series. Blackwell Science, 1989

[33] Van-Driest E R. The problem with aerodynamic heating. Aeronaut. Engng Rev. 15, 26-41 (1956)

[34] Delery J. Shock wave/turbulent boundary layer interaction and its control. Prog. Aerosp. Sci. 22, 209-280 (1985)

[35] Monaghan R J. On the Behavior of Boundary Layers at Supersonic Speeds. I.A.S. - R A. S Proceedings, 1955

[36] Jeong J, Hussain F. On the identification of a vortex. J. Fluid Mech. 285, 69-94 (1995) 